\title{
Scale factor duality for conformal cyclic cosmologies
}

\author{
U. Camara da Silva, A.L. Alves Lima and G.M. Sotkov \\ Departamento de Física - CCE, \\ Universidade Federal de Espirito Santo, 29075-900, Vitoria ES, Brazil \\ E-mail: ulyssescamara@gmail.com, andrealves.fis@gmail.com, \\ gsotkov@yahoo.com.br
}

ABSTRACT: The scale factor duality is a symmetry of dilaton gravity which is known to lead to pre-big-bang cosmologies. A conformal time version of the scale factor duality (SFD) was recently implemented as a UV/IR symmetry between decelerated and accelerated phases of the post-big-bang evolution within Einstein gravity coupled to a scalar field. The problem investigated in the present paper concerns the employment of the conformal time SFD methods to the construction of pre-big-bang and cyclic extensions of these models. We demonstrate that each big-bang model gives rise to two qualitatively different prebig-bang evolutions: a contraction/expansion SFD model and Penrose's Conformal Cyclic Cosmology (CCC). A few examples of SFD symmetric cyclic universes involving certain gauged Kähler sigma models minimally coupled to Einstein gravity are studied. We also describe the specific SFD features of the thermodynamics and the conditions for validity of the generalized second law in the case of Gauss-Bonnet (GB) extension of these selected CCC models.

KeYwords: Cosmology of Theories beyond the SM, Space-Time Symmetries, Classical Theories of Gravity

ARXIV EPRINT: 1604.08032 


\section{Contents}

1 Introduction 1

2 Pre-big-bang evolution from SFD 4

2.1 SFD-symmetric pre-big-bang extensions 4

2.2 Dual conformal diagrams 5

2.3 Cyclic SFD extensions 8

3 Scale factor duality for matter fields $\quad 9$

3.1 Dual and partially self-dual fluids $\quad 9$

$\begin{array}{lll}3.2 & \text { Dual scalar fields } & 11\end{array}$

4 SFD symmetric conformal cyclic cosmologies $\quad 14$

$\begin{array}{lll}4.1 & \text { Conformal crossover } & 14\end{array}$

$\begin{array}{lll}4.2 & \text { Conformal cyclic cosmology } & 15\end{array}$

$\begin{array}{lll}4.3 & \text { CCC vs. SFD - Matter transformations } & 16\end{array}$

$\begin{array}{lll}4.4 \text { CCC vs. SFD - Suppressed rest-mass conditions } & 19\end{array}$

4.5 CCC and SFD - Comparison 21

5 On the thermodynamics of SFD symmetric CCC models 22

$\begin{array}{lll}5.1 & \text { SFD thermodynamics } & 22\end{array}$

5.2 SFD for horizons thermodynamics 24

$\begin{array}{lll}5.3 & \text { Gauss-Bonnet extension and crossover entropy } & 27\end{array}$

6 Concluding remarks $\quad 29$

6.1 More on contraction/expansion SFD models 30

$\begin{array}{lll}6.2 & \text { On the scale factor duality of adiabatic fluctuations } & 31\end{array}$

6.3 Towards the conformal crossover holography 34

$\begin{array}{ll}\text { A Cyclic SFD models } & 35\end{array}$

B Proof of the $2^{\text {nd }}$ law for modified Chaplygin gas models 36

\section{Introduction}

Recent accumulation of observational evidences [1] in favor of certain inflationary improvements of the $\Lambda \mathrm{CDM}$ model has stimulated the search for the symmetry principles governing the universe evolution [2-8]. An important advance has been reached in the construction of a few selected families of inflaton superpotentials (representing universal attractors [9-11]) within the frameworks of (conformal) supergravity [12, 13]. 
The theoretical interpretations of the new astrophysical data do not, however, completely rule out the eventual physical relevance of appropriately chosen "pre-big-bang" [14, 15] or "cyclic" [16-19] extensions of the standard $\Lambda$ CDM universe. In these models the universe exists previously to the big-bang, in a phase of accelerated or/and decelerated contraction or expansion. The transition between standard post-big-bang evolution and these pre-big-bang phases may be achieved by non-singular or singular bounces (leading to a big-crunch/big-bang "joint" [20]), or else by the conformal identification of the future space-like infinity of the "previous universe" with our big-bang singularity, as in the conformal cyclic cosmology (CCC) models [17]. The conceptual advantage of such models is that there is no beginning of time and the very special state seen at the big-bang arises as a result of the pre-big-bang evolution. The challenge consists in arranging this previous evolution in order to address all the problems that are usually solved by inflation, without introducing any inconsistencies with the null energy condition (NEC) and with the increasing of entropy, i.e. with the generalized second law. Within the vast variety of cyclic cosmological models, characterized by the big-crunch-to-big-bang joints, one should also stand out the cyclic extensions of the post-big-bang eternal (chaotic) inflationary models with appropriate matter potentials, admitting regions of negative values [19, 21].

Symmetry requirements of a "stringy" origin are known to be an important tool in the construction of pre-big-bang models $[14,15]$. They allow one to define extensions of the Friedmann-Robertson-Walker (FRW) solutions before the singularity, and to determine the explicit form of the pre-big-bang matter content: e.g., they fix the equation of state (EoS) of the fluid, or the potential of the corresponding inflaton or quintessence scalar fields before the big-bang. The most representative example of such symmetries is given by the scale factor duality (SFD) - an extension of the superstring T-duality [22] for time dependent cosmological backgrounds in dilaton gravity, realized as a combination of scale factor inversions together with time reflections, preserving the form of the Friedmann equations. It provides alternative pre-big-bang models that admit different cosmic and conformal time realizations, both in Einstein and in dilaton gravities [14, 23-26].

The present paper is devoted to the further investigation of the cosmological applications of a conformal time scale factor duality [23], in FRW universes with metric

$$
d s^{2}=a^{2}(\eta)\left(-d \eta^{2}+\frac{d r^{2}}{1-K r^{2}}+r^{2} d \theta^{2}+r^{2} \sin ^{2} \theta d \varphi^{2}\right) .
$$

Here the spatial curvature is determined by $K=0, \pm 1$, we denote $a^{\prime}=\frac{d a}{d \eta}$, etc., in what follows, and use units in which $8 \pi G=1$. The duality transformations are composed of the scale-factor inversion

$$
\tilde{a}(\tilde{\eta})=c_{0}^{2} / a(\eta), \quad \text { with } \quad \tilde{\eta}= \pm \eta+\text { constant }
$$

followed by the specific transformation for the energy densities $\rho$ and $\tilde{\rho}$ and the pressures $p$ and $\tilde{p}$ of the matter fluids:

$$
\tilde{a}^{2} \tilde{\rho}(\tilde{a})=a^{2} \rho(a), \quad \tilde{a}^{2}[3 \tilde{p}(\tilde{a})+\tilde{\rho}(\tilde{a})]=-a^{2}[3 p(a)+\rho(a)]
$$


that may be combined into an expression for the equation of state parameters $w(\rho) \equiv p / \rho$ of the fluids, reading

$$
w(\rho)+\tilde{w}(\tilde{\rho})=-\frac{2}{3} .
$$

The duality acts in Einstein (and in Gauss-Bonnet) gravity, and is a symmetry of the space of solutions of the Friedmann equations

$$
\frac{1}{3} \rho=\left(\frac{a^{\prime}}{a^{2}}\right)^{2}+\frac{K}{a^{2}}, \quad p=\left(\frac{a^{\prime}}{a^{2}}\right)^{2}-\frac{2 a^{\prime \prime}}{a^{3}}-\frac{K}{a^{2}}, \quad \rho^{\prime}+3 \frac{a^{\prime}}{a}(\rho+p)=0 .
$$

If one knows the evolution of a FRW universe described by a set $\{a, \rho, w\}$, then one has automatically the solution of eqs. (1.3) for the 'dual' universe described by $\{\tilde{a}, \tilde{\rho}, \tilde{w}\}$ as well. As one can see directly from (1.2c), SFD maps accelerated periods into decelerated periods, and vice-versa - fluids such as radiation or dark matter, for which $w>-\frac{1}{3}$, are mapped into dark energy fluids with $w<-\frac{1}{3}$. Further, the weak energy condition is preserved for fluids with $-1 \leq w(\rho) \leq \frac{1}{3}$; that is, if $w(\rho)$ belongs to this interval, then so will $\tilde{w}(\tilde{\rho})$. On the other hand, the dual of a fluid with $w>\frac{1}{3}$ will be a phantom fluid with $\tilde{w}<-1$, so SFD also provides the possibility of describing phantom fluids from the knowledge of the behavior of non-phantom matter. Recent investigation [23] of the self-duality aspects of the conformal time scale factor duality has demonstrated that it can play an important role in the description of the post-big-bang phases of the universe evolution, acting as a UV/IR symmetry relating the final (almost de Sitter) stage of the universe with its (radiation filled) beginning, and also determining the form of the matter self-interaction.

The problem we address concerns the construction of pre-big-bang and cyclic universes using conformal time SFD (1.2) as a symmetry principle. That is, given a model of postbig-bang cosmic evolution then, following refs. [14, 15], its pre-big-bang phase will be defined by the corresponding dual universe. This is equivalent to implement the most relevant features of the pre-big-bang models of dilaton gravity, but here in its Einsteinframe version and replacing the dilaton with an appropriate self-interacting scalar field, as suggested in ref. [23]. Consider as an example the flat $\Lambda$ CDM model and its dual:

$$
\rho=\frac{\rho_{r}}{a^{4}}+\frac{\rho_{d}}{a^{3}}+\rho_{\Lambda}, \quad \tilde{\rho}=\frac{\tilde{\rho}_{r}}{\tilde{a}^{4}}+\frac{\tilde{\rho}_{d w}}{\tilde{a}}+\tilde{\rho}_{\Lambda},
$$

with $\rho_{\Lambda}=\Lambda$ being a positive cosmological constant, and $\rho_{r}$ and $\rho_{d}$ representing radiation and (barionic plus dark) matter, respectively. The dual, pre-big-bang universe is filled with another cosmological constant, radiation and a gas of domain walls represented by $\tilde{\rho}_{d w}$, with the relative densities fixed, according to the first of eqs. (1.2b), as

$$
\rho_{r} / \tilde{\rho}_{\Lambda}=\tilde{\rho}_{r} / \rho_{\Lambda}=\left(\rho_{d} / \tilde{\rho}_{d w}\right)^{2}=c_{0}^{4} .
$$

Our main goal is to describe how, although with the same dual matter content, SFD naturally gives rise to two qualitatively different types of pre-big-bang evolutions. The first one is obtained from scale factor inversions combined with conformal time translations, and it consists of a collapsing universe which ends in a decelerated phase leading to a big-crunch 
identified with the big-bang of the subsequent expanding evolution. This is similar in spirit to the ekpyrotic cosmologies $[18,20]$ and to the original Veneziano E-frame pre-big-bang models [27]. In the second possibility, scale factor inversion is combined with conformal time reflections. Then both the post- and the pre-big-bang phases are expanding universes: one ends at an accelerated expansion followed by the big-bang of the other, as in the Conformal Cyclic Cosmologies of ref. [17].

The construction of pre-big-bang phases derived from SFD is developed in section 2. In section 3 we elaborate on the effect of SFD in matter fields, in particular its description in terms of a self-interacting scalar field, and introduce the concept of duality and partial self-duality between the matter fluids filling two consecutive aeons. The similarities and the differences between SFD cosmologies and CCC, together with the restrictions needed to construct SFD symmetric models compatible with CCC, are presented in section 4 . It is also shown that the SFD symmetry, when applied to CCC models, selects a family of cosmological models whose matter content coincides with the $\mathrm{S} U(1,1) / \mathrm{U}(1)$ gauged Kähler sigma models with a specific self-interaction. In section 5 we derive the consistency conditions imposed on the generalized second law of thermodynamics in the case of the Gauss-Bonnet extension of these cyclic cosmological models. Our concluding section 6 is mainly devoted to the discussion of the consequences of the scale factor duality on the properties of the adiabatic fluctuations of the SFD symmetric FRW backgrounds, with also some comments on the holographic features of the conformal crossover.

\section{Pre-big-bang evolution from SFD}

Let $\check{a}(\check{\eta})$ be the scale factor of a flat $(K=0)$ expanding universe with a big-bang. The SFD transformations gives us a dual universe. We shall describe now what are the allowed behaviors of the dual scale factor $\hat{a}(\hat{\eta})$ with the dual conformal time $\hat{\eta}$.

\subsection{SFD-symmetric pre-big-bang extensions}

The SFD transformation of the energy density, eq. (1.2b), when combined with the Friedmann equations result in ${ }^{1}$

$$
\check{a} \check{H}= \pm \hat{a} \hat{H}
$$

Hence the dual of an expanding universe, with $\check{H}>0$, may be either another expanding universe, with $\hat{H}>0$, or a collapsing one, with $\hat{H}<0$. It is eq. (2.1), together with the inversion of the scale factor, that fixes the relation between $\check{\eta}$ and $\hat{\eta}$ to be linear: choosing $\check{\eta}=0$ for some $\check{a}_{0}$ and following the positions of the signs, we have

$$
\check{\eta}=\int_{\check{a}_{0}}^{\check{a}} \frac{d \check{a}}{\check{a}} \frac{1}{\check{a} \check{H}}=\mp \int_{c_{0}^{2} / \check{a} 0}^{\hat{a}} \frac{d \hat{a}}{\hat{a}} \frac{1}{\hat{a} \hat{H}}=\mp \hat{\eta}+\text { constant. }
$$

Thus we arrive at the conclusion that, for a given post-big-bang universe, the scale factor inversions give rise to two kinds of pre-big-bang extensions: (i) pairs of expanding/expanding

\footnotetext{
${ }^{1}$ The "checks and hats" notation adopted in this paper follows Penrose [17]. The mimics a future lightcone, and the^a past lightcone, therefore their usage for post- and pre-big-bang evolutions, respectively.
} 
(henceforth abbreviated as ' $\mathrm{E} / \mathrm{E}$ ') dual universes that are related by a conformal time reflection plus a translation and (ii) pairs of contracting/expanding (' $\mathrm{C} / \mathrm{E}$ ') dual universes, related by a simple time translation.

It is convenient to place the big-bang, $\{\check{a}=0\}$, at $\check{\eta}=0$. Then in the case of $\mathrm{E} / \mathrm{E}$ universes, eq. (2.2) becomes $\check{\eta}=-\hat{\eta}$ and one can further drop the marks on the $\eta$ 's to write the scale factor inversion (1.2a) as

$$
\check{a} \check{H}=\hat{a} \hat{H}, \quad \check{a}(\eta)=\frac{c_{0}^{2}}{\hat{a}(-\eta)} .
$$

For a pair of $\mathrm{C} / \mathrm{E}$ universes, the contracting one does not have a big-bang. Instead, it begins with $\hat{a}=\infty$ (at a de Sitter "vacuum") and collapses to a big-crunch. We may place both singularities at $\check{\eta}=\hat{\eta}=0$, for

$$
\check{\eta}=\int_{0}^{\check{a}} \frac{d \check{a}}{\check{a}^{2} \check{H}}=\int_{0}^{\hat{a}} \frac{d \hat{a}}{\hat{a}^{2} \hat{H}}-\int_{0}^{\infty} \frac{d \hat{a}}{\hat{a}^{2} \hat{H}}=\hat{\eta}+\eta_{f},
$$

where $\eta_{f}$ is the conformal time duration of the universe:

$$
\eta_{f} \equiv \int_{\infty}^{0} \frac{d \hat{a}}{\hat{a}^{2} \hat{H}}=\int_{0}^{\infty} \frac{d \check{a}}{\check{a}^{2} \check{H}}
$$

So here the scale factor inversion (1.2a) takes a form

$$
\check{a} \check{H}=-\hat{a} \hat{H}, \quad \check{a}(\eta)=\frac{c_{0}^{2}}{\hat{a}\left(\eta-\eta_{f}\right)} .
$$

A late-time acceleration of the post-big-bang universe, as it happens in the $\Lambda$ CDM model, renders the conformal time duration $\eta_{f}$ finite. As a consequence of eq. (2.4), the corresponding dual universe also has the same finite conformal lifetime, and begins at $-\eta_{f}$ with $\hat{a}\left(-\eta_{f}\right)=0$ or $\hat{a}\left(-\eta_{f}\right)=\infty$ in the expanding or contracting cases, respectively. ${ }^{2}$ This provides the following picture. As the time parameter $\eta$ evolves, what we get is a whole history of an expanding or contracting universe $\{\hat{a}, \hat{\rho}, \hat{p}\}$ for $\eta \in\left[-\eta_{f}, 0\right]$ which is then followed by a big-bang and by a dual universe $\{\check{a}, \check{\rho}, \check{p}\}$ evolution as $\eta$ runs forward in $\left[0, \eta_{f}\right]$. Following Penrose [17], we will call each of these two "consecutive cosmological histories" an aeon. ${ }^{3}$

\subsection{Dual conformal diagrams}

The effect of the SFD transformation in the conformal diagrams sheds some insight into how the causal structures of dual universes are related. The Penrose diagram of a flat FRW universe is obtained by the usual coordinate transformation $(\eta, r) \mapsto(\tau, \chi)$,

$$
\begin{aligned}
& \eta=\frac{1}{2} \sec \left(\frac{\chi+\tau}{2}\right) \sec \left(\frac{\chi-\tau}{2}\right) \sin \tau ; \\
& r=\frac{1}{2} \sec \left(\frac{\chi+\tau}{2}\right) \sec \left(\frac{\chi-\tau}{2}\right) \sin \chi,
\end{aligned}
$$

\footnotetext{
${ }^{2}$ As mentioned above, SFD maps accelerated into decelerated phases. Thus for a $\mathrm{C} / \mathrm{E}$ pair, the phases will be mapped in "reversed order", i.e. one universe has a late-time and the other an early-time accelerated phase. This is depicted in figure 1.

${ }^{3}$ Note that, although "consecutive", both histories are eternal in cosmic time, e.g. as $\eta \rightarrow 0-$, cosmic time diverges, $\hat{t} \rightarrow \infty$. Thus the name "aeon".
} 


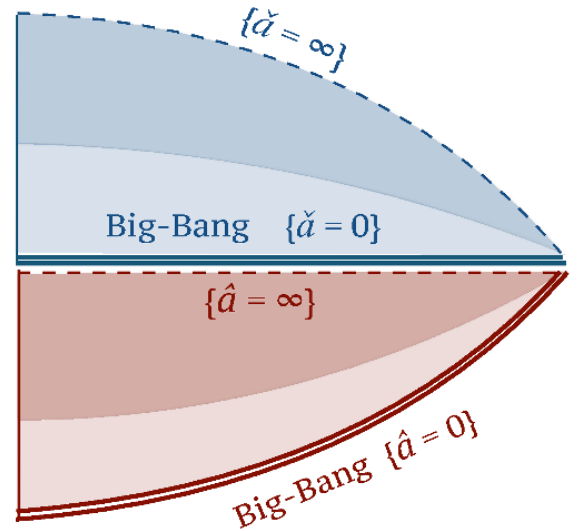

(a)

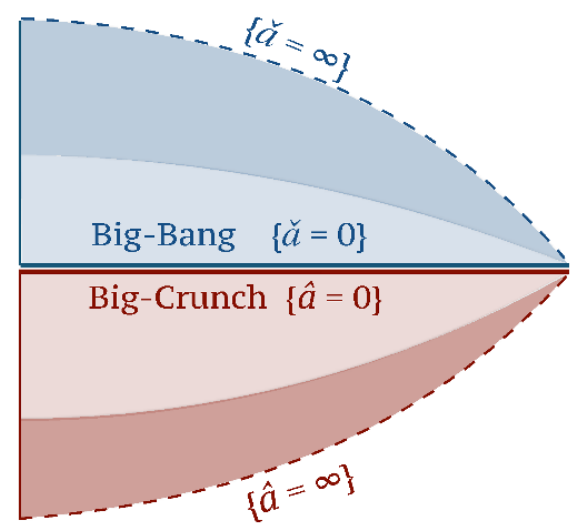

(b)

Figure 1. Conformal diagram for two consecutive dual universes with two periods of acceleration; (a) expanding/expanding and (b) contracting/expanding. Red and blue portions depict pre- and post-big-bang phases, respectively. Singularities are indicated by double lines, and infinities by dashed lines. Acceleration is positive in dark patches and negative in light ones.

which takes the (flat) FRW line element into

$$
d s^{2}=\frac{1}{4} a^{2}(\eta) \sec ^{2}\left(\frac{\chi+\tau}{2}\right) \sec ^{2}\left(\frac{\chi-\tau}{2}\right) d s_{\text {Einst }}^{2},
$$

where $d s_{\text {Einst }}^{2}=-d \tau^{2}+d \chi^{2}+\sin ^{2} \chi\left(d \theta^{2}+\sin ^{2} \theta d \varphi^{2}\right)$ is the metric of the Einstein universe (a 4-cylinder). The patch on the Einstein cylinder covered by a given FRW universe is bordered by the surfaces corresponding to the zeroes and infinities of the conformal factor multiplying $d s_{\text {Einst }}^{2}$ above.

If we choose the origin of conformal time such that the big-bang is placed at $\eta=0$, we see that the SFD transformation (2.3) relating E/E universes, $\hat{\eta}=-\check{\eta}$, is equivalent to $\hat{\tau}=-\check{\tau}$. Also, the inversion of the scale factor maps singularities into conformal infinities and vice-versa. The overall effect is that, given a Penrose diagram, its dual will be the mirrored image over the invariant line $\check{\tau}=\hat{\tau}=0$, with the roles of the lines corresponding to singularities and infinities interchanged. This can be seen, e.g. in figure 1(a). Thus we map initial into final conditions for the Friedmann equations, and vice-versa. The nullcone structure is, of course, mapped into itself. But note that a future null-cone in a universe is mapped into a past null-cone in its dual. In particular, this means that the particle and event horizons are mapped into each other (cf. [23]). This fact may be derived explicitly by writing the particle and event horizon radii, respectively, $\eta_{p}(a)=\int_{0}^{a} \frac{d a}{a^{2} H}$ and $\eta_{h}(a)=\int_{a}^{\infty} \frac{d a}{a^{2} H}$, as functions of the scale fator, then using eq. (2.3) to find

$$
\check{\eta}_{p}=\hat{\eta}_{h}, \quad \text { and } \quad \check{\eta}_{h}=\hat{\eta}_{p}
$$

An interesting consequence of eqs. (2.8) is to show how if the big-bang at $\{\check{a}=0\}$ is spacelike - a characteristic of decelerated expansion - , then so is the future infinity $\{\hat{a}=\infty\}$ of the dual universe - a characteristic of accelerated expansion, as it was to be expected. 




(a)

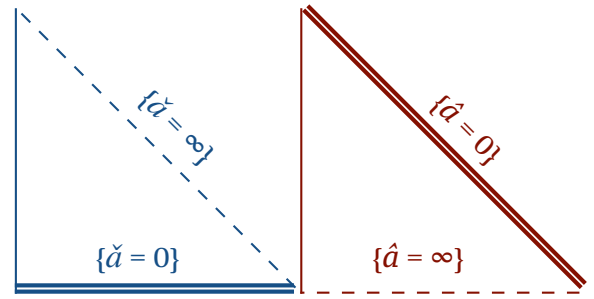

(b)

Figure 2. Causal diagrams for dual universes filled with fluids with constant equation of state parameters. The blue diagrams represent decelerated expansion $\left(\check{w}>-\frac{1}{3}\right)$ and have only a particle horizon. The dual red diagrams are both accelerating $\left(\hat{w}<-\frac{1}{3}\right)$. (a) Expansion/Expansion: the dual red diagram has accelerating expansion and only an event horizon. (b) Expansion/Contraction: the red diagram has accelerated contraction and only a particle horizon.

For a pair of $\mathrm{C} / \mathrm{E}$ dual universes we do not have the $\tau \mapsto-\tau$ mapping, and thus there is no "flipping" of the conformal diagram. We may, however, as has been noted above, choose the origin of conformal times such that conformal infinity and the dual bigbang coincide. The scale factor inversion, nevertheless, still exchanges the roles of these asymptotic surfaces. The overall effect for a universe with a finite conformal lifetime can be seen in figure 1(b). As to what regards the horizons, we have now: $\eta_{p}=\int_{\infty}^{a} d a\left(a^{2} H\right)^{-1}$ and $\eta_{h}=\int_{a}^{0} d a\left(a^{2} H\right)^{-1}$, which leads us to conclude that $\check{\eta}_{p}=\hat{\eta}_{p}$. A similar calculation is valid for event horizons $\check{\eta}_{h}=\hat{\eta}_{h}$. Therefore the particle horizons of the expanding and the contracting universes are mapped into each other, and so are the respective event horizons.

We shall be concerned in this paper with universes like the ones appearing in figure 1, in which there are two different phases of acceleration (such as in the $\Lambda$ CDM model), thus possessing both kinds of horizon and marked by a finite total conformal time duration $\eta_{f}=\int_{0}^{\infty} d a\left(a^{2} H\right)^{-1}=\eta_{p}+\eta_{h}$. This may, of course, not be always the case. For example, universes filled with a single perfect fluid with constant equation of state parameter $\check{w}>$ $-\frac{1}{3}$, and their duals, with $\hat{w}<-\frac{1}{3}$, have, each, only one kind of horizon as shown in figure 2 . Then either $\eta_{p}$ or $\eta_{h}$ are infinite, resulting in an infinite $\eta_{f}$ so eq. (2.5), for example, has to be modified. The crucial point here is this: when both boundary surfaces $\{a=0\}$ and $\{a=\infty\}$ are space-like, as in figure 1, we may identify the coinciding boundaries of consecutive aeons as one single space-like hypersurface $\mathcal{X}$, which we shall call a 'crossover surface'. It is conformally equivalent to the Euclidean 3-dimensional space (that is, a constant-time-surface in Minkowski space-time), and with our choice of conformal time, $\mathcal{X}=\{\eta=0\}$. Now in the case of figure 2, one cannot define properly a transition surface because this would amount to identify hypersurfaces with different signatures. For example, in a $\mathrm{C} / \mathrm{E}$ pair the contracting universe ends in a null hypersurface while the 
expanding universe begins in a space-like big-bang (figure 2(b)). As for the transition in a $\mathrm{E} / \mathrm{E}$ pair, it may be well defined but it can be done only once; that is, it existis only for "two consecutive aeons", and one cannot naturally build a "chain" of aeons, as it will be done presently in section 2.3 .

\subsection{Cyclic SFD extensions}

The rather unusual realization of the transition between expanding/expanding SFD universes - with the future infinity of one aeon being identified with the initial big-bang of the next - suggests that the pattern should repeat itself indefinitely. That is, it is natural to define a sequence of aeons, each being related to its following neighbor by an SFD transformation. We denote by

$$
\mathcal{A}_{j}=\left\{a_{j}\left(\eta_{j}\right) ; \quad \eta_{j} \in\left[(j-1) \eta_{f}, j \eta_{f}\right] ; \quad j \in \mathbb{Z}\right\}
$$

each aeon in the sequence. See figure 3. We thus partition the real line $\eta \in \mathbb{R}$ into a set of intervals $\left[(j-1) \eta_{f}, j \eta_{f}\right]$, with the same length, which are the domains of the scale factors $a_{j}\left(\eta_{j}\right)$ : we have $a_{j}\left(j \eta_{f}\right)=\infty$ and $a_{j}\left((j-1) \eta_{f}\right)=0$. Note that the order of the indices $j$ follow the positions of the "ends of the universe", e.g. $\mathcal{A}_{2}$ terminates at $\eta=2 \eta_{f}, \mathcal{A}_{1}$ at $\eta=1 \eta_{f}, \mathcal{A}_{0}$ at $\eta=0$, etc. Note also that, by construction and as it should be, according to eq. (2.4), every aeon $\mathcal{A}_{j}$ in the sequence has the same conformal "lifespan" $\eta_{f}$.

Our construction depends upon $\eta_{f}$ being finite. As discussed in the preceding section 2.2, this implies that the surfaces $\mathcal{X}_{j} \equiv\left\{\eta=j \eta_{f}\right\}$ are 3 -dimensional space-like crossover surfaces between the aeons. Alternativelly,

$$
\mathcal{X}_{j} \equiv\left\{a_{j}\left(j \eta_{f}\right)=\infty \approx a_{j+1}\left(j \eta_{f}\right)=0\right\} .
$$

See figure 3(a).

The scale factor $a_{j}\left(\eta_{j}\right)$ of each aeon determines the scale factor of the next one via the SFD transformation, which reads

$$
a_{j+1}\left(\eta_{j+1}\right)=\frac{c_{0}^{2}}{a_{j}\left(-\eta_{j+1}+2 j \eta_{f}\right)} ; a_{j-1}\left(\eta_{j-1}\right)=\frac{c_{0}^{2}}{a_{j}\left(-\eta_{j-1}+2(j-1) \eta_{f}\right)} .
$$

Accordingly, the densities and pressures of the fluids are related by the periodic analogs of eqs. (1.2a), (1.2b) and (1.2c):

$$
\begin{aligned}
a_{j}^{2} \rho_{j}=a_{j+1}^{2} \rho_{j+1}, \quad a_{j}^{2}\left(3 p_{j}+\rho_{j}\right) & =-a_{j+1}^{2}\left(3 p_{j+1}+\rho_{j+1}\right), \\
w_{j}+w_{j+1} & =-\frac{2}{3} .
\end{aligned}
$$

Eqs. (2.9)-(2.11) define the sequence of aeons we have proposed. Regardless of the infinite number of aeons, this cyclic SFD model consists of a repetition of the same dual pair $\left\{a_{j}, a_{j+1}=c_{0}^{2} / a_{j}\right\}$. Indeed, it is an important consequence of the "cyclic $Z_{2}$ form" of the scale factor inversions in (2.10), that $a_{j-1}=a_{j+1}$, as can be seen in figure 3(b). Naturally, the matter content presents analogous relations, with $\rho_{j-1}=\rho_{j+1}$ and $p_{j-1}=p_{j+1}$, while $\rho_{j}$ is related to as $\rho_{j+1}=\frac{a_{j}^{4}}{c_{0}^{4}} \rho_{j}$, etc. An explicit example of our construction is given in appendix A. 


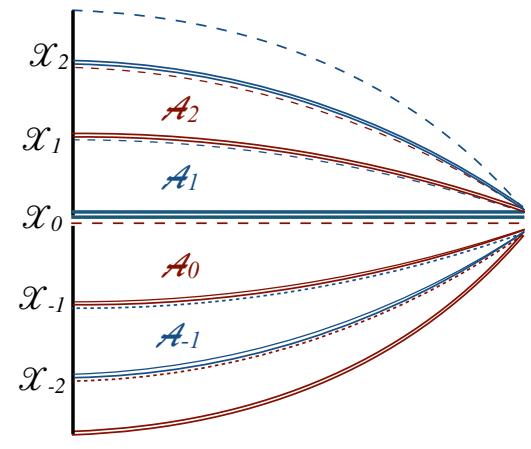

(a)

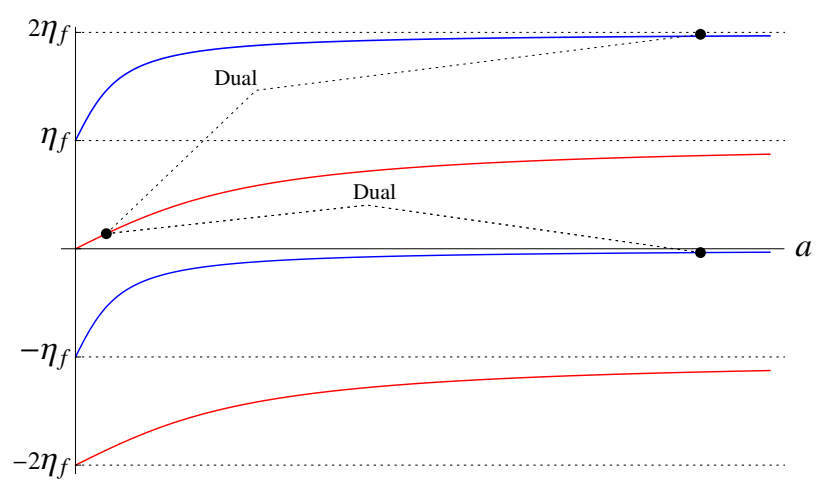

(b)

Figure 3. Sequences of aeons. Same colors indicate aeons with identical matter and scale factor. (a) Conformal diagram representing the sequence with its space-like crossover surfaces $\mathcal{X}_{j}$; double lines indicate a singularity $\left(a_{j}=0\right)$, and dashed lines a conformal infinity $\left(a_{j}=\infty\right)$. (b) The conformal time evolution of the scale factors, as given implicitly by $\eta_{j}\left(a_{j}\right)$. The black dots mark a sequence of dual instants related by SFD according to eq. (2.10).

\section{Scale factor duality for matter fields}

The matter content of the SFD-symmetric pre-big-bang cosmologies consists of pairs of barotropic fluids or, alternatively, pairs of self-interacting scalar fields. The pre- and postbig-bang equations of state of the fluids, as well as the corresponding scalar field potentials, are related by the transformations (1.2b). In this section we investigate the most general consequences and restrictions imposed by these extension rules on the matter constituents. We are mainly interested on their interrelations, such as $\hat{\rho}(\check{\rho})$ and $\hat{\sigma}(\check{\sigma})$.

\subsection{Dual and partially self-dual fluids}

According to the requirement of SFD symmetry, for each given post-big-bang density $\check{\rho}(\check{a})$, one can easily determine the form of its dual,

$$
\hat{\rho}\left(\hat{a} ; \hat{\rho}_{J}\right)=\frac{1}{c_{0}^{4}} \check{a}^{4} \check{\rho}\left(\check{a} ; \check{\rho}_{J}\right) .
$$

We have introduced explicitly here the collections of relative densities parameters $\check{\rho}_{J}$ and $\hat{\rho}_{J}$, because (3.1) implies relations between them and the SFD parameter $c_{0}$. For example, the $\Lambda \mathrm{CDM}$ parameters, $\rho_{J} \equiv\left\{\rho_{r}, \rho_{d}, \rho_{\Lambda}\right\}$, relate to their dual as in eq. (1.5). We shall consider a special class of cosmological models satisfying an additional partial self-duality condition ${ }^{4}$ by requiring that the forms of the fluid densities and pressures (and of their EoS) are preserved by SFD transformations, but allowing changes in the values of the fluid's parameters $\hat{\rho}_{J} \neq \check{\rho}_{J}$. For example, a universe with radiation and cosmological constant is partially self-dual since, if $\check{\rho}=\check{\rho}_{r} \check{a}^{-4}+\check{\rho}_{\Lambda}$, then from (3.1) the dual aeon has the same constituents: $\hat{\rho}=\hat{\rho}_{\Lambda}+\hat{\rho}_{r} \hat{a}^{-4}$, but with different relative densities $\check{\rho}_{J}$ and $\hat{\rho}_{J}$, which are

\footnotetext{
${ }^{4}$ It is similar (but not identical) to the partial self-duality concept introduced in section 3 of ref. [28], within the framework of 3D New Massive Gravity Holography.
} 
related by $\check{\rho}_{r} / \hat{\rho}_{\Lambda}=c_{0}^{4}=\hat{\rho}_{r} / \check{\rho}_{\Lambda}$. We note that partial self-duality is a weaker version of the more restrictive condition of (complete) self-duality introduced in [23]. The latter is realized by further demanding $\hat{\rho}_{J}=\check{\rho}_{J}$, in which case both aeons are completely identical. ${ }^{5}$

A particularly relevant family of two interacting fluids for pre-big-bang extensions is given by the modified Chaplygin gas model [29-31]

$$
\rho=\left(\rho_{\Lambda}^{\delta}+\rho_{r}^{\delta} a^{-4 \delta}\right)^{\frac{1}{\delta}}, \quad p=\frac{1}{3} \rho-\frac{4 \rho_{\Lambda}^{\delta}}{3} \rho^{1-\delta}, \quad 0<\delta \leq 1 .
$$

We have chosen to study the cyclic extensions for a subclass of models determined by the restriction $0<\delta \leq 1$. The upper limit for $\delta$ comes from the requirement that the corresponding curvatures $R(\delta)=2 \rho_{\Lambda}^{\delta}(\rho)^{1-\delta}$ are monotonically decreasing functions of the density $\infty \geq \rho \geq \rho_{\Lambda}$. The lower limit $\delta>0$ selects matter fluids which give rise to a finite conformal time lifespan, $\eta \in\left(0, \eta_{f}\right)$, and behave asymptotically as a cosmological constant for $a \rightarrow \infty$ and as radiation when $a \rightarrow 0$. By construction they imply the existence of big-bang singularity followed by a decelerated and then an accelerated period of expansion in the post-big-bang phase. ${ }^{6}$ Acceleration changes sign (i.e. vanishes) at a critical value of the scale factor, $a=a_{c r}$, or, alternatively, a critical value of the energy density, $\rho=\rho_{c r}$, such that

$$
a_{c r}=\left(\frac{\rho_{r}}{\rho_{\Lambda}}\right)^{\frac{1}{4}}, \quad \rho_{c r}=2 \rho_{\Lambda},
$$

when the deceleration parameter $q(a) \equiv \frac{1}{2}\left(1+3 \frac{p}{\rho}\right)$ vanishes. The modified Chaplygin models are in fact the only ones to behave asymptotically as a cosmological constant within a very general class of partially self-dual fluids [23], which makes them particularly useful in the construction of SFD symmetric CCC models, realized in section 4 below. Their conformal lifetime is given by:

$$
\eta_{f}=\frac{\sqrt{3}}{4 \delta\left(\rho_{r} \rho_{\Lambda}\right)^{\frac{1}{4}}} \frac{\left[\Gamma\left(\frac{1}{4 \delta}\right)\right]^{2}}{\Gamma\left(\frac{1}{2 \delta}\right)},
$$

as one can easily confirm from the analytic form [23] of the (implicit) scale factor $\eta=\eta(a)$ evolution. Details concerning a particular example of $\delta=1 / 2$ model and its cyclic extension are presented in appendix A.

The relation between the parameters $\rho_{J}=\left\{\rho_{r}, \rho_{\Lambda}\right\}$ for two dual aeons is fixed by (3.1) to be

$$
c_{0}^{4}=\check{\rho}_{r} / \hat{\rho}_{\Lambda}=\hat{\rho}_{r} / \check{\rho}_{\Lambda},
$$

which keeps invariant (3.4); i.e. $\check{\eta}_{f}=\hat{\eta}_{f}=\eta_{f}$ as was to be expected. The dual pre-big-bang evolution also contains both periods, with the SFD transformations mapping accelerated

\footnotetext{
${ }^{5}$ E.g. the only self-dual perfect fluid, with a constant EoS parameter $w$, is the string gas model with $w=-1 / 3$ and $\rho(a)=\rho_{\text {str }} / a^{2}$. For a self-dual composition of two fluids, see ref. [23].

${ }^{6}$ Notice that one of its SFD dual geometries describe a contracting universe with monotonically increasing curvature starting from the initial de Sitter state of $\rho_{\Lambda}$ and reaching the bing-crunch singularity at $\rho \rightarrow \infty$.
} 
into decelerated phases and vice-versa, as discussed in section 1. The simple form the fluid density (3.2) allows us to derive from eq. (3.1) a relation between the post- and pre-big-bang energy densities:

$$
\hat{\rho}^{\delta}-\hat{\rho}_{\Lambda}^{\delta}=\frac{\hat{\rho}_{\Lambda}^{\delta} \check{\rho}_{\Lambda}^{\delta}}{\check{\rho}^{\delta}-\check{\rho}_{\Lambda}^{\delta}},
$$

which shows explicitly how the SFD transformations act on the matter content exchanging initial and final conditions. For example, the duality between the scale factors $\check{a}=0$ and $\hat{a}=\infty$ at $\hat{\eta}=0=\check{\eta}$ relates $\check{\rho}(0)=\infty$ to $\hat{\rho}(\infty)=\hat{\rho}_{\Lambda}$ and vice-versa. The above high-to-low densities transformation ${ }^{7}$ highlights the intrinsic UV/IR nature of conformal time scale factor duality.

We have to also mention another important feature of every partially self-dual SFD model, and in particular of the modified Chaplygin gas (3.2), namely their invariance under another conformal SFD transformation, acting now within each aeon $\mathcal{A}_{j}$, that maps the early-time decelerated phase into the late-time accelerated phase as follows:

$$
\tilde{a}_{j}\left(\eta_{j}\right)=\frac{c_{0 c}^{2}(j)}{a_{j}\left(\eta_{f}-\eta_{j}\right)}, \quad c_{0 c}^{4}(j)=\frac{\rho_{r}^{(j)}}{\rho_{\Lambda}^{(j)}}=\left(\frac{\rho_{d}^{(j)}}{\rho_{d w}^{(j)}}\right)^{2} .
$$

Observe that here we have a (very specific) combination of time reflection and translation (with a fixed point $\eta_{c}=\eta_{f} / 2$ ). The composition of the two different SFD transformations - one acting within each aeon with the intrinsic parameter $c_{0 c}^{2}(j)$, followed by the interaeonic SFD given by eqs. (2.10) and (2.11) - defines a double SFD $Z_{2} \times Z_{2}$ symmetry. It is expressed between two consecutive aeons as

$$
\tilde{\tilde{a}}(\eta)=\frac{\check{c}_{0 c}^{2}}{\check{a}\left(\eta_{f}-\eta\right)}=\frac{\check{c}_{0 c}^{2}}{c_{0}^{2}} \hat{a}\left(\eta-\eta_{f}\right)=\frac{\check{c}_{0 c}^{2} \hat{c}_{0 c}^{2}}{c_{0}^{2} \tilde{\hat{a}}(-\eta)}=\frac{c_{0}^{2}}{\hat{\hat{a}}(-\eta)} .
$$

The identity $c_{0}^{4}=\check{c}_{0 c}^{2} \hat{c}_{0 c}^{2}$, automatically satisfied by the parameters $c_{0}^{4}=\hat{\rho}_{r} / \check{\rho}_{\Lambda}=\check{\rho}_{r} / \hat{\rho}_{\Lambda}$, $\check{c}_{0 c}^{2}=\sqrt{\frac{\check{\rho}_{r}}{\check{\rho}_{\Lambda}}}$ and $\hat{c}_{0 c}^{2}=\sqrt{\frac{\hat{\rho}_{\Lambda}}{\hat{\rho}_{r}}}$, ensures the consistency of these double SFD transformations.

\subsection{Dual scalar fields}

We next consider the equivalent scalar field description of the pairs of dual fluids, and the SFD transformations between them. Each of the pre- and post-big-bang fluids can be replaced by corresponding scalar fields $\hat{\sigma}$ and $\check{\sigma}$, with potentials $\check{V}\left(\check{\sigma} ; \check{\rho}_{J}\right)$ and $\hat{V}\left(\hat{\sigma} ; \hat{\rho}_{J}\right)$, such that

$$
\check{\rho}=\frac{1}{2}\left(\check{\sigma}^{\prime} / \check{a}\right)^{2}+\check{V}(\check{\sigma}), \quad \check{p}=\frac{1}{2}\left(\check{\sigma}^{\prime} / \check{a}\right)^{2}-\check{V}(\check{\sigma}),
$$

and the same for $\hat{\sigma}, \hat{V}(\hat{\sigma})$, etc. Then the SFD transformations (1.2b) can be rewritten as

$$
\hat{V}(\hat{\sigma})=\frac{1}{3} \check{V}(\check{\sigma})+\frac{2}{3}\left(\check{\sigma}^{\prime} / \check{a}\right)^{2}, \quad\left(\hat{\sigma}^{\prime} / \hat{a}\right)^{2}=\frac{4}{3} \check{V}(\check{\sigma})-\frac{1}{3}\left(\check{\sigma}^{\prime} / \check{a}\right)^{2} .
$$

\footnotetext{
${ }^{7}$ Together with a similar duality relation for the curvatures, viz. $\hat{R}=2 \hat{\rho}_{\Lambda}^{\delta}(\hat{\rho})^{1-\delta}$ and $\check{R}=2 \check{\rho}_{\Lambda}^{\delta}(\check{\rho})^{1-\delta}$.
} 
Given the potential $\check{V}\left(\check{\sigma} ; \check{\rho}_{J}\right)$ of the post-big-bang model, our goal is to find the explicit form of the scalar fields duality transformation $\hat{\sigma}=\hat{\sigma}(\check{\sigma})$ and to use it further in the derivation of the corresponding dual or partially self-dual pre-big-bang potential $\hat{V}\left(\hat{\sigma} ; \hat{\rho}_{J}\right)$. The Friedmann equations (1.3), when combined with eqs. (3.9), lead to the auxiliary relations

$$
\left(\check{\sigma}^{\prime}\right)^{2}=-\frac{\check{a}^{3}}{3} \frac{d \check{\rho}}{d \check{a}}, \quad \check{V}(\check{a})=\check{\rho}+\frac{\check{a}}{6} \frac{d \check{\rho}}{d \check{a}}, \quad \frac{d \check{\sigma}}{d \check{a}}= \pm \sqrt{\frac{\check{a} \frac{d \check{\rho}}{3 K-\check{a}}}{2 \check{\rho}}}
$$

which allow to realize the scalar field as a function of the scale factor - i.e. given $\check{\rho}(\check{a})$ to find $\check{\sigma}(\check{a})$. In the cases when its inverse $\check{a}(\check{\sigma})$ can be obtained (and so $\check{\rho}(\check{\sigma})$ as well), one derives from eqs. (3.1), (3.9) and (3.10) the pre-big-bang potential and the scalar fields SFD transformations:

$$
\hat{V}(\hat{\sigma})=\frac{\check{a}^{4}(\check{\sigma})}{c_{0}^{4}}\left(\frac{4}{3} \check{\rho}(\check{\sigma})-\check{V}(\check{\sigma})\right) ; \quad \hat{\sigma}(\check{\sigma})= \pm \int \sqrt{\frac{3 \check{V}-\check{\rho}}{3(\check{\rho}-\check{V})}} d \check{\sigma}+\sigma_{0},
$$

where we have also used the scale factor inversions in the form: $\hat{a}(\hat{\sigma})=c_{0}^{2} / \check{a}(\check{\sigma})$. A partially self-dual fluid, naturally, gives rise to a partially self-dual potential, such that $\hat{V}\left(\hat{\sigma} ; \hat{\rho}_{J}\right)=\check{V}\left(\hat{\sigma} ; \hat{\rho}_{J}\right)$.

For the modified Chaplygin gas (3.2), the explicit solution for the the scale factor $a(\sigma)$ is given by [23]

$$
\check{a}(\check{\sigma})=\left(\frac{\check{\rho}_{R}}{\check{\rho}_{\Lambda}}\right)^{\frac{1}{4}}\left[\sinh \left(\delta\left(\sigma_{0}-\check{\sigma}\right)\right)\right]^{-\frac{1}{2 \delta}},
$$

with $\check{\sigma} \leq \sigma_{0}$, and a similar one for $\hat{a}(\hat{\sigma})$. Then, from eqs. (3.10), one can easily find $\hat{\sigma}(\check{\sigma})$,

$$
\sinh \left(\delta\left(\hat{\sigma}-\sigma_{0}\right)\right)=\frac{1}{\sinh \left(\delta\left(\check{\sigma}-\sigma_{0}\right)\right)},
$$

and the corresponding partially self-dual potential

$$
\hat{V}(\hat{\sigma})=\frac{\hat{\rho}_{\Lambda}}{3}\left\{\left[\cosh ^{2}\left(\delta\left(\hat{\sigma}-\sigma_{0}\right)\right)\right]^{\frac{1}{\delta}}+2\left[\cosh ^{2}\left(\delta\left(\hat{\sigma}-\sigma_{0}\right)\right)\right]^{\frac{1-\delta}{\delta}}\right\} .
$$

Due to the difference between the vacuum densities of the two aeons, $\hat{\rho}_{\Lambda} \neq \check{\rho}_{\Lambda}$, the dual fields $\hat{\sigma}$ and $\check{\sigma}$ have different masses related by

$$
\hat{m}_{\hat{\sigma}}^{2}=\frac{\hat{\rho}_{\Lambda}}{\check{\rho}_{\Lambda}} \check{m}_{\check{\sigma}}^{2}, \quad \hat{m}_{\hat{\sigma}}^{2}=\hat{V}^{\prime \prime}\left(\hat{\sigma}=\sigma_{0}\right)=2 \delta(3-2 \delta) \frac{\hat{\rho}_{\Lambda}}{3} .
$$

Since the potential (3.15) is symmetric under reflections about $\sigma_{0}$, we may choose $\sigma_{0}=0$ and to further consider its "left side" only, i.e. $\sigma<0$. Then as a consequence eq. (3.14), both $\check{\sigma}$ and $\hat{\sigma}$ will run on this chosen side. There is an expanding solution for the scale factor (3.13), with the field $\hat{\sigma}$ rolling from the big-bang at $V(\infty)=\infty$, down to the de Sitter vacuum at $V(0)=\rho_{\Lambda}$. Its SFD dual solution, obtained from eq. (2.5), describes a contracting universe, starting at the vacuum, where the field climbs the potential towards 


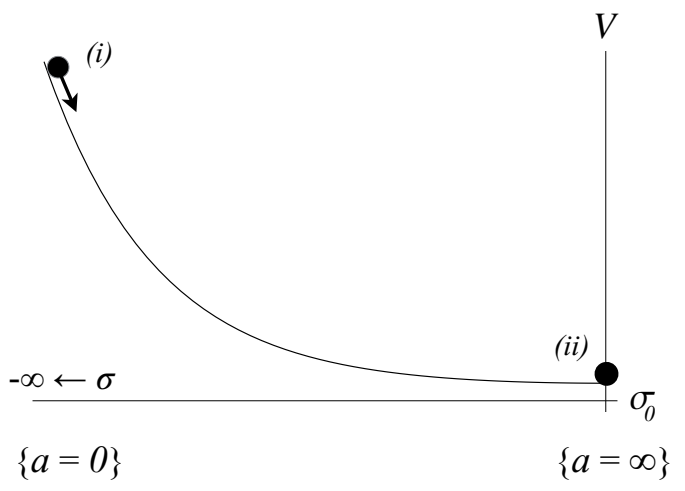

(a)

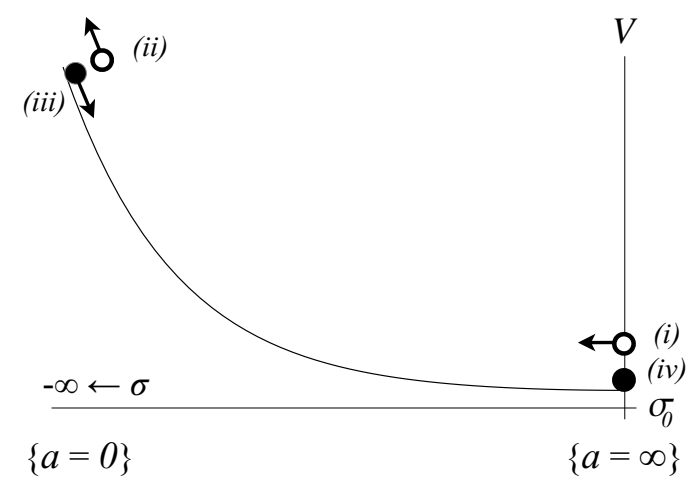

(b)

Figure 4. The possible pre-big-bang evolutions of $\sigma$ associated with the potential (3.15). (a) E/E case: both dual fields roll down the potential towards the de Sitter vacuum. (b) C/E case: the pre-big-bang field $\hat{\sigma}$ (white ball) begins at the vacuum and rolls up to the big-crunch; then the dual post-big-bang field $\check{\sigma}$ (black ball) rolls down from the big-bang to de Sitter.

a big-crunch. Let us assume that $\check{\sigma}$ represents an expanding solution. If we differentiate eq. (3.14), we find that

$$
\frac{d \check{\sigma}}{d \check{\eta}}=-\left(\frac{\cosh \delta \hat{\sigma}}{\cosh \delta \check{\sigma} \sinh ^{2} \delta \hat{\sigma}}\right) \frac{d \hat{\eta}}{d \check{\eta}} \frac{d \hat{\sigma}}{d \hat{\eta}}
$$

The expression in parenthesis is always positive, therefore we can have two different cyclic extensions, of the $\mathrm{E} / \mathrm{E}$ or $\mathrm{C} / \mathrm{E}$ type, as presented in figure 4 . For simplicity, we now take $V(\sigma)$ to be "completely self-dual", i.e. $\check{\rho}_{\Lambda}=\hat{\rho}_{\Lambda}$, so that both dual universes may be described with one single potential.

If $d \hat{\eta} / d \check{\eta}<0$, the field $\hat{\sigma}$ and its dual $\check{\sigma}$ roll down ${ }^{8}$ the potential in both universes. This corresponds to an E/E dual pair for which, indeed, $d \hat{\eta} / d \check{\eta}=-1$, as seen in section 2 (cf. eq. (2.3)). The behavior of the fields is symbolically depicted on figure 4(a). Here, the de Sitter vacuum is conformally identified with the big-bang at the crossover surface, viz. $\{\check{\sigma}=0\} \approx\{\hat{\sigma}=-\infty\} \approx \mathcal{X}$. In other words, when the field $\hat{\sigma}$ reaches the de Sitter vacuum, eq. (3.14) implies that its dual $\check{\sigma}$ "reappears" at the top of the potential and yields a new big-bang and a new expanding universe as it rolls down.

The other possibility has $d \hat{\eta} / d \check{\eta}>0$. This is a $\mathrm{C} / \mathrm{E}$ dual pair, for which $d \hat{\eta} / d \check{\eta}=+1$ (cf. eq. (2.5)). As depicted in figure 4(b), if $\check{\sigma}$ rolls down, so that $d \check{\sigma} / d \eta<0$ and the universe expands, then $\hat{\sigma}$ rolls up and the dual universe collapses. One may describe the evolution with (i) $\hat{\sigma}$ beginning in the de Sitter vacuum and (ii) $\hat{\sigma}$ climbing the potential towards the big-crunch, which is (iii) identified with the big-bang, and (iv) then $\check{\sigma}$ returns down to the vacuum. It is important to mention that this kind of evolution is similar to the weak-coupling version of pre-big-bang and cyclic models ${ }^{9}$ discussed in section 3 of

\footnotetext{
${ }^{8}$ Both could also climb the potential, leading to dual contracting universes, the time-reversal of the E/E pairs. We are, as always, not considering such cases.

${ }^{9}$ The similarity to the weak-coupling regime stems from our choice of $\sigma<0$.
} 
ref. [20]; it is, however, different from the original dilaton gravity strong-coupling prebig-bang scenario [15, 27]. Notice that (as it also happens in the E/E case) the SFD transformation (3.14) exchanges the "boundary conditions" of the scalar field, that is: the configuration (i) is dual to (iii), etc. Finally, note that the identification of steps (i) and (iv), and the endless repetition of the cycle just described is straightforward. A few applications of these results will be discussed in section 6 .

\section{SFD symmetric conformal cyclic cosmologies}

The pairs of scale factor dual FRW solutions $(\hat{a}, \hat{\rho}, \hat{p})$ and $(\check{a}, \check{\rho}, \check{p})$, joined at their time limits $\left(\eta=0\right.$ and $\left.\eta= \pm \eta_{f}\right)$, define pre-big-bang and cyclic cosmological models that might suffer of certain inconsistencies related to discontinuities of the scale factor derivatives. ${ }^{10}$ One expects to overcome the eventual "gluing problems" by an appropriate choice of their matter content or else by calling for quantum or string corrections to make them smooth. The conceptually different nature of the transition region in the expanding/expanding universes offers however another option.

\subsection{Conformal crossover}

As we have shown in section 2, the conformal crossover consists in the identification of the asymptotic space-like 3D surface, representing "de Sitter-like" future infinity of the past aeon with the big-bang singularity of the present aeon. In other words we identify the poles of $\hat{a} \approx 1 / \eta$ with the zeros of $\check{a} \approx \eta$ and more generally $\hat{a}^{2} \approx \eta^{1+3 w} \approx 1 / \check{a}^{2}$, as required by scale factor duality (2.3). Hence the description of such "conformal crossover" junction involves a pair of metrics belonging to the conformal (Weyl) equivalence class of transition metrics, known to be the hallmark of the conformal cyclic cosmologies [17, 32]. In fact the asymptotic behavior of SFD expanding/expanding universes is nothing but a particular realization of the Penrose's "reciprocal hypothesis"

$$
\Omega \omega=-1, \quad d \check{s}^{2}=\omega^{2} d s^{2}, \quad d \hat{s}^{2}=\Omega^{2} d s^{2}, \quad d s^{2}=-d \eta^{2}+d \mathbf{x}^{2},
$$

that indicates how to choose one finite at $\eta=0$ (i.e. without zeros and poles) representative of this equivalence class of metrics. Since by construction we have that $\omega$ vanishes and $\Omega$ diverges at $\eta=0$, therefore eq. (4.1) ensures that $d s^{2} \propto \Omega^{2} d \check{s}^{2}=\omega^{2} d \hat{s}^{2}$ remains finite. The negative sign in eq. (4.1) is required for $\omega$ to be a function with non-zero derivative which can be extended to the "opposite" aeon continuously: it simply vanishes at the big-bang and then becomes negative. Assuming that the space at the transition region is homogeneous and isotropic one can easily relate "reciprocal hypothesis" (4.1) to the scale factor inversions (2.3)

$$
\omega(\eta)=\left\{\begin{array}{ll}
\frac{1}{c_{0}} \check{a}(\eta) \\
-\frac{1}{c_{0}} \check{a}(-\eta)
\end{array} \quad \text { and } \quad \Omega(\eta)= \begin{cases}-\frac{1}{c_{0}} \hat{a}(-\eta), & \eta>0 \\
\frac{1}{c_{0}} \hat{a}(\eta), & \eta<0\end{cases}\right.
$$

\footnotetext{
${ }^{10}$ Similar to the "graceful exit" problems of the pre-big-bang models of dilaton gravity [15] and of the more general contracting/expanding cosmologies as well.
} 


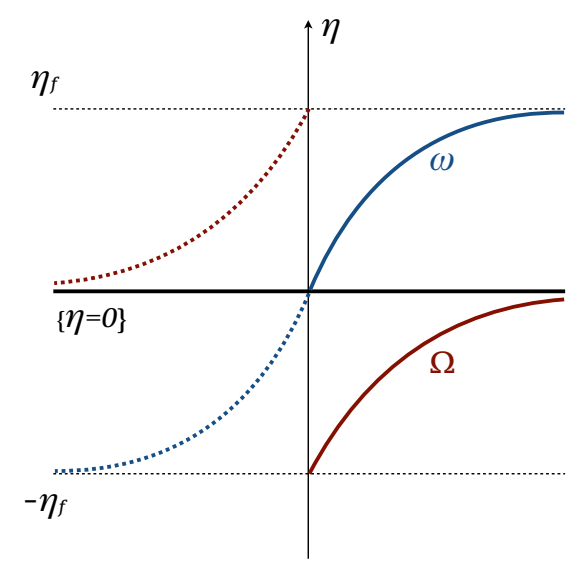

Figure 5. The conformal factors across the infinity/big-bang surface. The blue line gives $\omega(\eta)$ and the red lines give $\Omega(\eta)$; the dotted portions indicate the extensions to the opposite aeon.

as shown on figure 4.1. It gives for the conformal factors the appropriate form at their respective aeons, where they are proportional to the scale factors (e.g. $\omega^{2}(\eta) \sim \check{a}^{2}(\eta)$ for $\eta>0)$. The change of sign in the arguments at the "opposite" aeons (e.g. $\omega^{2}(\eta) \sim \check{a}^{2}(-\eta)$ for $\eta<0)$ are necessary since the functions $\check{a}(\eta)$ and $\hat{a}(\eta)$ are only defined, each respectively, at the disjoint intervals $\eta \in\left[0, \eta_{f}\right)$ and $\eta \in\left[-\eta_{f}, 0\right)$. Thus eq. (4.2) provides the most natural "extension" of both metrics (4.1) to the whole interval $\left[-\eta_{f},+\eta_{f}\right]$.

\subsection{Conformal cyclic cosmology}

The relation between the "reciprocal hypothesis" (4.1) and conformal time scale factor duality (2.3) suggests that an appropriate implementation of SFD as a symmetry of conformal cyclic cosmologies might take place. In order to make such a "CCC/SFD correspondence" more precise, we find worthwhile to briefly stand out the main CCC characteristics. According to the standard cosmological model the fate of our universe is to expand at an accelerated rate forever and become cold and empty. Penrose further assumes [17] that in the very far future (almost) all the matter will be swallowed by black holes and these, in turn, will completely evaporate. ${ }^{11}$ The massive particles which eventually escape falling into a black hole will, by then, have lost their rest mass due to an "anti-Higgs" mechanism resulting in spontaneous enhancement of the symmetries (see [17] for a more detailed discussion). The universe will then be filled only with cold radiation and, therefore conformally (Weyl) invariant. On the other hand, the early universe, shortly after the big-bang, is also conformally invariant, filled with hot radiation, so one may conformally transform the old universe into the early one and vice-versa. The conformal identification is done, not for the beginning and the end of one same universe, but for two consecutive eternal universes, each named an 'aeon'. Thus the big-bang singularity of an aeon and the conformal infinity of its predecessor are identified at one single surface $\mathcal{X}$. The metrics of each aeon, close to this surface $\hat{g}_{\mu \nu}=\Omega g_{\mu \nu}$ and $\check{g}_{\mu \nu}=\omega g_{\mu \nu}$ must satisfy the reciprocal hypothesis (4.1). Notice however that the pair of CCC metrics $\left(\hat{g}_{\mu \nu}, \check{g}_{\mu \nu}\right)$ are not

\footnotetext{
${ }^{11}$ Due to "supercooling" bellow the temperature of thermal equilibrium with Hawking radiation.
} 
in general of FRW type and therefore the conformal factor inversion in CCC is only an asymptotic symmetry that also keeps untouched the space-time causal structure. In the transition "crossover" region where this symmetry is valid, "there is no one metric", but an equivalence classes of metrics and as a consequence, there is no notion of scales, distances or of a "cosmic time". Therefore within the frameworks of the Penrose's CCC concepts for Universe evolution the massless matter, the radiation and the gravitational waves are eternal and everpresent, e.g. freely transiting between the consecutive aeons and the only effect of their bypassing through the crossover surfaces $\mathcal{X}$ is the change in their frequencies, amplitudes, energies, etc. reflecting the conformal principle that at $\mathcal{X}$ 's all the distances, momenta, etc. are in fact equivalent.

Another distinctive feature of CCC is the so-called 'suppressed rest-mass hypothesis' (SRM). It ensures the intrinsic consistency and continuity at $\eta=0$ for the derivatives of the conformal factors, representing the two (oppositely oriented ) normal vectors $\check{N}_{\mu}=\check{\gamma} \partial_{\mu} \Omega$ and $\hat{N}_{\mu}=\hat{\gamma} \partial_{\mu} \omega$ to the surface $\mathcal{X}=\{\omega=0\}$. Written in terms of $\omega$ it reads ${ }^{12}$

$$
\begin{aligned}
\hat{N}_{\mu} \hat{N}^{\mu} & =\hat{\gamma}^{2} \partial_{\mu} \omega \partial^{\mu} \omega=-1+(2-Q) \omega^{2}+O\left[\omega^{3}\right], & \left.\check{N}_{\mu} \check{N}^{\mu}\right|_{\mathcal{X}}=-1=\left.\hat{N}_{\mu} \hat{N}^{\mu}\right|_{\mathcal{X}}, \\
\hat{\gamma} & =\sqrt{\frac{3}{c_{0}^{2} \hat{\rho}_{\Lambda}}}=\sqrt{\frac{3 c_{0}^{2}}{\check{\rho}_{r}}}=\check{\gamma}, & c_{0}^{4}=\frac{\hat{\rho}_{r}}{\check{\rho}_{\Lambda}}=\frac{\check{\rho}_{r}}{\hat{\rho}_{\Lambda}}
\end{aligned}
$$

where $Q$ is an "universal constant" to depend upon the matter content of both aeons and on the details of mass generation mechanism in the new aeon as well (see the appendix A of ref. [32], as well as ref. [33]). In fact eq. (4.3) imposes the correct initial/final conditions at $\eta=0$ needed to guarantee that the unit normals remain time-like and their norms are close to unity up to second order terms in the vicinity of $\mathcal{X}$. The missing linear term is a specific requirement, forbidding that non-relativistic matter (dust) $\rho_{d} \approx 1 / \hat{a}^{3}$ to contribute in the neighbors of crossover $\mathcal{X}$; it is closely related to the initial conditions for the Yamabe equation $[17,32,33]$, assumed to govern the matter induced by the conformal factor $\Omega$ in the present aeon.

The above mentioned CCC requirements assure the consistency of crossover transition and the desired matter content that provides the asymptotic conformal symmetry. However they do not exhaust all the conditions that CCC must obey. As emphasized by Penrose [17] the most important ingredient of the Conformal Cyclic Cosmology concerns its thermodynamical (TD) properties - the definition of their entropy and the compatibility of the second law with the cyclic nature of the CCC evolution. We postpone the discussion of the some of thermodynamical aspects of CCC models and the problem concerning TD consistency of their SFD symmetric versions to section 5 below.

\subsection{CCC vs. SFD - Matter transformations}

The scale factor inversion (2.3) can be easily recognized as a particular discrete $Z_{2}$ Weyl transformation $\Omega(a)$ relating the metric of two consecutive aeons:

$$
d \check{s}^{2}=\Omega^{-4} d \hat{s}^{2}, \quad \hat{a}=\Omega^{2}(\check{a}) \check{a}, \quad \Omega^{2}(\check{a})=\frac{c_{0}^{2}}{\check{a}^{2}},
$$

\footnotetext{
${ }^{12}$ Notice the difference between Penrose's signature $(+---)$, the opposite of ours. Also, in refs. [17, 32] the authors use a different normalization of the vectors $\check{N}_{\mu}, \hat{N}_{\mu}$, namely with $\check{\gamma}=1=\hat{\gamma}$, which corresponds to fixing $\hat{\rho}_{\Lambda}=3=\check{\rho}_{\Lambda}, c_{0}^{2}=1$.
} 
as one can see from eqs. (4.2). Notice, however, the important difference: while the SFDsymmetric CCC models are invariant under such a restricted Weyl transformation (4.4) within the whole interval $\left[-\eta_{f},+\eta_{f}\right]$, the original CCC proposal [17] manifests an even more general conformal (Weyl) invariance, but in the transition region $\eta \approx 0$ only.

This observation allows to relate the matter content of the two aeons, by adapting the SFD methods described in section 3 to the case of CCC models. The "field" responsible for this mechanism is the conformal factor $\Omega$ of one aeon, when extended into the opposite one. For any conformal transformation of the metric, like $\check{g}_{\mu \nu}=\Omega^{-4} \hat{g}_{\mu \nu}$, the Einstein tensor $\check{G}_{\mu \nu}=\check{R}_{\mu \nu}-\frac{1}{2} \check{R} \check{g}_{\mu \nu}$ transforms as

$$
\hat{G}_{\mu \nu}=\check{G}_{\mu \nu}+2 \Upsilon_{\mu} \Upsilon_{\nu}-2 \check{\nabla}_{\mu} \Upsilon_{\nu}+2 \check{g}_{\mu \nu} \check{g}^{\alpha \beta} \check{\nabla}_{\alpha} \Upsilon_{\beta}+\check{g}_{\mu \nu} \check{g}^{\alpha \beta} \Upsilon_{\alpha} \Upsilon_{\beta},
$$

where $\Upsilon_{\mu} \equiv 2 \partial_{\mu} \log \Omega$. Such transformations, considered as symmetries of the pre-big-bang SFD universe (and of the crossover region of generic CCC models), have to preserve the form of the Einstein equations at both aeons

$$
\hat{G}_{\mu \nu}=\hat{T}_{\mu \nu} \text { and } \quad \check{G}_{\mu \nu}=\check{T}_{\mu \nu}
$$

with $\check{T}_{\mu \nu}$ and $\hat{T}_{\mu \nu}$ appropriately related. Therefore, we must incorporate the extra terms at the right-hand-side of eq. (4.5) into the stress-tensor, reading the transformation of the Einstein tensor as an effective transformation between the stress tensors of the present and past aeons:

$$
\begin{aligned}
\hat{T}_{\mu \nu} & =\check{T}_{\mu \nu}+t_{\mu \nu}, \quad \text { where } \\
t_{\mu \nu} & =\frac{4}{\phi^{2}} \check{\nabla}_{\mu} \phi \check{\nabla}_{\nu} \phi+\frac{4}{\phi} \check{\nabla}_{\mu} \check{\nabla}_{\nu} \phi-\left(\frac{4}{\phi} \check{\square} \phi-\frac{8}{\phi^{2}} \check{g}^{\alpha \beta} \check{\nabla}_{\alpha} \phi \check{\nabla}_{\beta} \phi\right) \check{g}_{\mu \nu}, \quad \text { with } \quad \phi \equiv 1 / \Omega
\end{aligned}
$$

The tensor $t_{\mu \nu}$ represents the contribution to $\hat{T}_{\mu \nu}$ from the Weyl rescaling of the metric $\check{g}_{\mu \nu}$ of its dual universe. It is then natural to consider such pairs of aeons as being different descriptions of the same universe seen from two non-equivalent conformal frames. Within the frameworks of Conformal Cyclic Cosmology the above results also suggest that certain gravitational degree of freedom ${ }^{13}$ (encoded in $\Omega$ ) is transmitted from one aeon to the matter content of the consecutive aeon, via the peculiar double-role of the "gravitational/matter field" $\phi[34]$.

The SFD symmetric CCCs possess an important new feature - although the conformal symmetry is broken, they remain invariant under a residual discrete Weyl transformation (4.4). It acts as an $U V / I R$ duality symmetry relating the large scales behavior (at late times) of one aeon to the small scales (at earlier times) of its dual and vice-versa. Thus, given the past aeon fluid density, it allows to determine the matter content of the present aeon. In order to derive the SFD counterpart of CCC's stress-tensor transformations we replace $\phi=\frac{1}{c_{0}} \check{a}(\eta)$ into eqs. (4.7). As expected, the result of this substitution reproduces the matter fluid SFD transformations (1.2b): the 00-component is simply $\check{a}^{2} \check{\rho}=\hat{a}^{2} \hat{\rho}$, while from the $i i$-components we get the pressure transformation $\check{a}^{2}(3 \check{p}+\check{\rho})=-\hat{a}^{2}(3 \hat{p}+\hat{\rho})$.

\footnotetext{
${ }^{13}$ They are not, however, "pure gauge" degrees of freedom, since the local conformal (Weyl) symmetry is broken outside of the transition region between the two aeons.
} 
In order to highlight the CCC features of the SFD extensions of modified Chaplygin gas cosmology (3.2), we have to further implement the "reciprocal hypothesis" (4.1) and of the asymptotic Weyl symmetry (in the neighborhood of $\mathcal{X}$ ) for the pair $(\hat{\sigma}, \check{\sigma})$ of matter fields as well. As it was shown in section 3 , the corresponding partially self-dual matter Lagrangians $\hat{\mathcal{L}}\left(\hat{\sigma}, \hat{\rho}_{J}\right)$ and $\check{\mathcal{L}}\left(\check{\sigma}, \check{\rho}_{J}\right)$ contain "almost identical" potentials (3.15), with different masses $\hat{m}_{\hat{\sigma}}^{2} \neq \check{m}_{\tilde{\sigma}}^{2}$, that are related by the SFD transformations (3.14), (3.12) and (3.16). The expanding/expanding nature of the CCC evolution implies that we have to restrict the values of the scalar fields, say $-\infty<\hat{\sigma} \leq 0$, thus considering only "the expanding halves" of the potentials $\hat{V}$ and $\check{V}$ and "gluing" them at $\mathcal{X}$ by conformal identification of the "de Sitter vacua final state" $\hat{\sigma}_{v a c}=0$ of the past aeon with the "initial big-bang state" $\check{\sigma}_{b b}=-\infty$ of the present aeon. Notice that the SFD mapping realized around the conformal crossover is transforming the accelerated dark energy dominated phase $\hat{\sigma}_{c r} \leq \hat{\sigma} \leq 0$ (i.e. small $\hat{\sigma}$ values $)^{14}$ of the past aeon into the decelerated radiation dominated phase $-\infty<\check{\sigma} \leq \check{\sigma}_{c r}$ (i.e. large $\check{\sigma}$ values) of the present aeon and vice versa. The consistent SFD description of the conformal crossover requires to further extend the conformal (Weyl) equivalence class of the transition metrics to include the matter fields as well. The problem is that the SFD transformation (3.14) of the pair of canonical scalar fields $(\hat{\sigma}, \check{\sigma})$ has not the desired Weyl form. One can use the fact that the Weyl transformations for the scale factor are well defined in the conformal time frame (see also eq. (2.6a)) as a guide of how to convert the $\check{\sigma}(\hat{\sigma})$ transformation into a standard Weyl form by an appropriate change of the fields variables $\sigma \rightarrow \Phi$, such that the infinite range of values of $\sigma \in(-\infty, \infty)$ is mapped into a finite interval for $\Phi \in\left[-\Phi_{0}, \Phi_{0}\right]$. An important hint is given by the following equivalent forms of the conformal factor $\Omega(\check{a})$ and SFD matter transformations (for $\sigma_{0}=0$ ):

$$
\Omega^{2}(\check{a})=\frac{c_{0}^{2}}{\check{a}^{2}}=\sqrt{\frac{\check{\rho}_{\Lambda}}{\hat{\rho}_{\Lambda}}}(\sinh (\delta \check{\sigma}))^{\frac{1}{\delta}}, \quad\left(\frac{\hat{\rho}_{\Lambda}}{\check{\rho}_{\Lambda}}\right)^{\delta} \tanh ^{2}(\delta \hat{\sigma})=\Omega^{-2 \delta}\left(\frac{\check{\rho}_{\Lambda}}{\hat{\rho}_{\Lambda}}\right)^{\delta} \tanh ^{2}(\delta \check{\sigma}),
$$

obtained from eqs. (3.13) and (3.14). As suggested in ref. [23], it is then natural to introduce a new field variable having however a non-canonical kinetic term:

$$
\begin{aligned}
\hat{\Phi}^{2}=\hat{\phi} \hat{\phi} & =6\left(\frac{\hat{\rho}_{\Lambda}}{\check{\rho}_{\Lambda}}\right)^{\delta} \tanh ^{2}(\delta \hat{\sigma}), \quad \check{\Phi}^{2}=\check{\phi} \bar{\phi}=6\left(\frac{\check{\rho}_{\Lambda}}{\hat{\rho}_{\Lambda}}\right)^{\delta} \tanh ^{2}(\delta \check{\sigma}), \\
& -\sqrt{6}\left(\frac{\rho_{\Lambda}}{\hat{\rho}_{\Lambda}}\right)^{\delta / 2} \leq \hat{\Phi} \leq 0, \quad 0 \leq \check{\Phi} \leq \sqrt{6}\left(\frac{\hat{\rho}_{\Lambda}}{\check{\rho}_{\Lambda}}\right)^{\delta / 2} .
\end{aligned}
$$

In fact, the substitution (4.9) transforms the self-dual Chaplygin gas model (3.15) into the "gauged" $\mathrm{S} U(1,1) / \mathrm{U}(1)$ Kähler sigma model minimally coupled to Einstein gravity

$$
\hat{K}=-\frac{1}{2 \delta^{2}} \ln \left(1-\frac{\check{\rho}_{\Lambda}^{\delta}}{6 \hat{\rho}_{\Lambda}^{\delta}} \hat{\phi} \overline{\hat{\phi}}\right), \quad \hat{V}(\hat{\Phi})=\frac{\hat{\rho}_{\Lambda}^{2-\delta}}{9}\left(\frac{6}{6 \hat{\rho}_{\Lambda}^{\delta}-\hat{\Phi}^{2} \check{\rho}_{\Lambda}^{\delta}}\right)^{\frac{1}{\delta}}\left(9 \hat{\rho}_{\Lambda}^{\delta}-\hat{\Phi}^{2} \check{\rho}_{\Lambda}^{\delta}\right)
$$

with the gauge fixing of $U(1)$ symmetry realized as $\hat{\phi}=\overline{\hat{\phi}}=\hat{\Phi}$, and the same for the dual fields $\check{\phi}$. We have adopted the standard Kähler sigma model notations:

$$
\hat{\mathcal{L}}_{\text {matter }}(\hat{\phi}, \overline{\hat{\phi}})=g^{\hat{\phi} \hat{\phi}} \hat{\nabla}_{\mu} \hat{\phi} \hat{\nabla}^{\mu} \overline{\hat{\phi}}-\hat{V}(\hat{\Phi}), \quad g^{\hat{\phi} \hat{\phi}}=\partial_{\hat{\phi}} \partial_{\bar{\phi}} \hat{K},
$$

\footnotetext{
${ }^{14}$ Where $\hat{\sigma}_{c r}=\frac{1}{2 \delta} \ln (1+\sqrt{2})=\check{\sigma}_{c r}$ is determined as a zero acceleration value of the scalar field, $q\left(\sigma_{c r}\right)=0$.
} 
where, due to the specific choice of the Kähler potential $\hat{K}(\hat{\phi}, \overline{\hat{\phi}})$ in eq. (4.10), the resulting Kähler metric $g^{\phi \bar{\phi}}$ has a constant curvature $R_{K}=-4 \delta^{2}$. Within the framework of the above "Kähler representation" of CCC model, the new matter fields SFD transformation takes the proper Weyl form we were seeking:

$$
\hat{\Phi}=\Omega^{-2 \delta} \check{\Phi}, \quad \hat{a}=\Omega^{2}(\check{a}) \check{a}, \quad \Omega^{2}(\check{\Phi})=\frac{c_{0}^{2}}{\check{a}^{2}}=\left(\frac{\check{\Phi}^{2} \check{\rho}_{\Lambda}^{\delta}}{6 \check{\rho}_{\Lambda}^{\delta}-\check{\Phi}^{2} \hat{\rho}_{\Lambda}^{\delta}}\right)^{\frac{1}{2 \delta}} .
$$

Let us mention one "unexpected SUSY feature" of this Kähler sigma model realization of SFD-symmetric CCC (4.11) discovered in ref. [23]: it turns out to be identical with the bosonic part of the $\mathcal{N}=1$ supergravity with one chiral matter supermultiplet [12,35] and

a very special form of its matter superpotential $|\hat{\mathcal{Z}}|=\sqrt{\frac{\hat{\rho}_{\Lambda}}{3}} e^{\delta \hat{K}}=\sqrt{\frac{2}{3} \hat{\rho}}$, that gives rise to the partially self-dual potential (4.10).

The above discussion of the CCC properties of the pre-big-bang extension of modified Chaplygin gas model (3.2) has demonstrated the advantages of the SFD methods in the selection of the matter fields self-interactions as well as in the description of their behavior both around the conformal crossover and far from it. We have also accumulated several evidences that its Kähler form appears to be a promising candidate for SFD-symmetric CCC with physically relevant matter content of a certain supergravity origin. It remains, however, to verify whether it also satisfies all the other CCC conditions [17].

\subsection{CCC vs. SFD - Suppressed rest-mass conditions}

The SFD counterpart of Penrose's SRM hypothesis can be obtained by replacing the SFD form (4.2) of the conformal factor $\omega$ into eq. (4.3), which now reads:

$$
\begin{array}{ll}
\check{\gamma}^{2} \partial_{\mu} \omega \partial^{\mu} \omega=-1+\frac{(2-Q)}{c_{0}^{2}} \check{a}^{2}(\eta)+O\left[\check{a}^{3}(\eta)\right], & \text { for } \quad \eta>0, \\
\hat{\gamma}^{2} \partial_{\mu} \omega \partial^{\mu} \omega=-1+c_{0}^{2}(2-Q) \hat{a}^{-2}(\eta)+O\left[\hat{a}^{-3}(\eta)\right], & \text { for } \quad \eta<0 .
\end{array}
$$

On the other hand, by using once again eq. (4.2) for $\omega$, we can directly calculate the norms of the vectors $\check{N}_{\mu}=\check{\gamma} \partial_{\mu} \Omega$ and $\hat{N}_{\mu}=\hat{\gamma} \partial_{\mu} \omega$,

$$
\begin{aligned}
& \check{\gamma}^{2} \partial_{\mu} \omega \partial^{\mu} \omega=-\frac{3}{\check{\rho}_{r}}\left(\check{a}^{\prime}(\eta)\right)^{2}=-\frac{1}{\check{\rho}_{r}} \check{a}^{4} \check{\rho}, \quad \text { for } \quad \eta>0, \\
& \hat{\gamma}^{2} \partial_{\mu} \omega \partial^{\mu} \omega=-\frac{3}{\hat{\rho}_{\Lambda}}\left(\frac{\hat{a}^{\prime}(\eta)}{\hat{a}^{2}(\eta)}\right)^{2}=-\frac{1}{\hat{\rho}_{\Lambda}} \hat{\rho}, \quad \text { for } \quad \eta<0 .
\end{aligned}
$$

At the last step of these equalities we have used the Friedmann equations. Note that both the norms of $\check{N}_{\mu}$ and $\hat{N}_{\mu}$ turn out to be proportional to the density of the first aeon $\hat{\rho}=\frac{\check{a}^{4}}{c_{0}^{4}} \check{\rho}$. The identification of the r.h.s. of eqs. (4.13) and (4.14) furnishes the explicit SFD form of the original SRM condition (4.3).

When the expansion (4.13) contains only integer power of the scale factor, we may include the terms up to fourth order,

$$
\check{\gamma}^{2} \partial_{\mu} \omega \partial^{\mu} \omega=-1+\frac{(2-Q)}{c_{0}^{2}} \check{a}^{2}-\frac{\check{Q}_{3}}{c_{0}^{2}} \check{a}^{3}-\frac{\check{Q}_{4}}{c_{0}^{2}} \check{a}^{4}+\cdots \quad \text { for } \quad \eta>0
$$


and accordingly for $\eta<0$, so the implementation of the SRM condition on SFD models may be written as

$$
\begin{aligned}
& \check{\rho}=\frac{\check{\rho}_{r}}{\check{a}^{4}}+\frac{\check{\rho}_{s t r}}{\check{a}^{2}}+\frac{\check{\rho}_{d w}}{\check{a}}+\check{\rho}_{\Lambda}+\cdots, \quad \text { for } \quad \check{a} \rightarrow 0, \\
& \hat{\rho}=\hat{\rho}_{\Lambda}+\frac{\hat{\rho}_{s t r}}{\hat{a}^{2}}+\frac{\hat{\rho}_{d}}{\hat{a}^{3}}+\frac{\hat{\rho}_{r}}{\hat{a}^{4}}+\cdots, \quad \text { for } \quad \hat{a} \rightarrow \infty .
\end{aligned}
$$

Thus, stoping at fourth order in the expansion (4.15), the universe is filled with a composition of perfect fluids, and the original SRM requirement that the term linear in $\omega$ must be excluded from the expansion in (4.13a) is, as expected, equivalent to the absence of a dust component in $\check{\rho}$ on the beginning of the the post-big-bang phase, i.e. $\check{\rho}_{d}=0$. Meanwhile, the requirement of normalization of $\check{N}_{\mu}$ and $\hat{N}_{\mu}$ imply that the dominating components, near $\mathcal{X}$, at the corresponding aeons are radiation $\left(\check{\rho}_{r} / \check{a}^{4}\right)$ and a cosmological constant $\hat{\rho}_{\Lambda}$. The second order term in (4.13a) turns out to be related to the presence in both aeons of a string gas component, with relative densities $\check{\rho}_{s t r}=\frac{\check{\rho}_{r}}{c_{0}^{2}}(Q-2)$ and $\hat{\rho}_{s t r}=c_{0}^{2} \hat{\rho}_{\Lambda}(Q-2){ }^{15}$ Similarly, $\check{Q}_{3}$ is related to a gas of domain walls $\left(\check{\rho}_{d w}\right)$, and $\check{Q}_{4}$ to a cosmological constant $\check{\rho}_{\Lambda}$ in the post-big-bang phase. We have

$$
\check{\rho}_{d w}=\frac{1}{c_{0}^{3}} \check{Q}_{3} \check{\rho}_{r}, \quad \check{\rho}_{\Lambda}=\frac{1}{c_{0}^{4}} \check{Q}_{4} \check{\rho}_{r} ; \quad \hat{\rho}_{d}=c_{0}^{3} \hat{Q}_{3} \hat{\rho}_{\Lambda}, \quad \hat{\rho}_{r}=c_{0}^{4} \hat{Q}_{4} \hat{\rho}_{\Lambda} .
$$

The coefficient $Q$ in eq. (4.3) is assumed by Penrose [32] to be "universal" in the sense that it is the same in avery aeon. If we assume this of the other coefficients, so that $\check{Q}_{3}=\hat{Q}_{3}$ and $\check{Q}_{4}=\hat{Q}_{4}$, then eqs. (4.17) fixes relations between ratios of densities in both aeons, viz.

$$
\hat{\rho}_{s t r} / \check{\rho}_{s t r}=c_{0}^{4}\left(\hat{\rho}_{\Lambda} / \check{\rho}_{r}\right), \quad \hat{\rho}_{d} / \check{\rho}_{d w}=c_{0}^{6}\left(\hat{\rho}_{\Lambda} / \check{\rho}_{r}\right), \quad \hat{\rho}_{r} / \check{\rho}_{\Lambda}=c_{0}^{8}\left(\hat{\rho}_{\Lambda} / \check{\rho}_{r}\right) .
$$

These are the same relations imposed by SFD with, also, $\hat{\rho}_{r} / \check{\rho}_{\Lambda}=c_{0}^{4}$ (cf. eq. (1.4)). We may then turn the argument around and conclude that SFD fixes $\check{Q}_{3}=\hat{Q}_{3}$ and $\check{Q}_{4}=\hat{Q}_{4}$.

The allowed presence of the dust component $\hat{\rho}_{d} / \hat{a}^{3}$ in the pre-big-bang phase stems from the fact that we are considering its "future asymptotic", and the SRM condition forbids the presence of dust in the "past asymptotic", i.e. near the big-bang. But we have been considering only one pair of dual aeons. We now turn to the implementation of the SRM condition on the cyclic extension composed of an infinite chain of aeons described in section 2.3. Then, the surface $\hat{a}=0$ is another crossover, in the vicinity of which $\hat{\rho}$ must satisfy an expansion of the same form of (4.16a), i.e. we must have $\hat{\rho}_{d}=0$ and this reflects on the following aeon, since eq. (4.18) then forces $\check{\rho}_{d w}=0$. Therefore the SRM condition, imposed on a chain of SFD aeons with perfect fluids, forbids both dust and its dual, a gas of domain walls.

This prohibition of even a late-time dust component moves us to consider models which are more elaborate than the simple composition of independent perfect fluids. The most

\footnotetext{
${ }^{15}$ Therefore the condition $Q=2$ obtained in ref. [33] is equivalent to the absence of a string gas. The result that the aeons are filled by perfect fluids as a consequence of an expansion around the crossover has also been found in [36].
} 
relevant here is the modified Chaplygin gas density (3.2), whose asymptotic limit near $\mathcal{X}$,

$$
\check{\rho}=\check{a}^{-4}\left(\check{\rho}_{r}^{\delta}+\check{\rho}_{\Lambda}^{\delta} \check{a}^{4 \delta}\right)^{1 / \delta}=\check{\rho}_{r} \check{a}^{-4}+\frac{\check{\rho}_{\Lambda}^{\delta}}{\delta \check{\rho}_{r}^{\delta}} \check{a}^{-4(1-\delta)}+O\left[\check{a}^{-4(1-2 \delta)}\right] \quad \text { for } \quad \check{a} \rightarrow 0
$$

is indeed "SRM consistent" if $\delta \in[1 / 2,1]$. If we also assume that the expansion is in integer powers of $\check{a}$, then the SRM condition selects three values of $\delta=\frac{1}{2}, \frac{3}{4}, 1$. The case with $\delta=\frac{3}{4}$ represents a particularly interesting SFD-symmetric CCC model, that manifests many of the properties of the Chaplygin-like quintessence models $[29,31]$ : at early times $(\check{a} \approx 0)$ it is approximated by radiation and domain walls $\check{\rho} \approx \check{\rho}_{r} / \check{a}^{4}+\check{\rho}_{d w} / \check{a}$, while at late times $(\check{a} \approx \infty)$ it gets a contribution from the cosmological constant and dust, $\check{\rho} \approx \check{\rho}_{\Lambda}+\check{\rho}_{d} / \check{a}^{3}$. In other words, it is an example of the desired cosmological model which has a late-time dust-like behavior while still respecting the SRM condition. Note that since every aeon in a SFD chain is filled with a Chaplygin gas with the same $\delta$ (but possibly different values of $\rho_{r}$ and $\rho_{\Lambda}$ ), the consistency of $\check{\rho}$ in eq. (4.19) above is sufficient to assure the consistency on every crossover of the chain. Note also that both the Chaplygin gas and the consistent composition of perfect fluids discussed above turn out to be partially self-dual.

When we consider the scalar field counterpart of the Chaplygin gas, the condition $\delta=\frac{1}{2}, \frac{3}{4}, 1$ fixes the curvature of the Kähler metric to be $R_{K}=-1,-\frac{9}{4},-4$. But the most important consequence of the SRM conditions is that they provide consistent initial and final conditions (on $\mathcal{X}$ ) not only for the scale factor $\hat{a}$ and its dual $\check{a}$, but for the pairs fields $(\hat{\sigma}, \check{\sigma})$ and $(\hat{\Phi}, \breve{\Phi})$ as well - it is enough to take into account eqs. (3.10), (3.13) and (4.9). In other words they guarantee the "crossover consistency" for the solutions of the matter fields equations of the considered partially self-dual massive $\mathrm{SU}(1,1) / \mathrm{U}(1)$ Kähler sigma model (4.11).

\subsection{CCC and SFD - Comparison}

Our investigation of the consequences of the implementation of scale factor duality to Penrose's CCC models has revealed certain similarities and diferences, in comparison with SFD-symmetric pre-big-bang and cyclic cosmologies. Their main common feature is the conformal crossover. In fact, both represent two different (but interrelated) implementations of the same symmetry principle of restricted and/or asymptotic conformal (Weyl) invariance. In SFD models, the scale factor inversion is an exact symmetry between the whole evolution of the universe background and its dual. It is, however, more restrictive then the CCC's asymptotic symmetries - SFD is a symmetry of the Friedmann equations only, and thus not valid when fluctuations are considered. Furthermore, it is not a "full" conformal transformation, but rather a particular discrete $Z_{2}$ subgroup of it. One consequence of these diferences is that SFD models satisfy rather rigid restrictions on the matter contents of the two aeons, while in the CCC models the "suppressed rest-mass" condition, although compatible with scale factor duality, turns out to be much less restrictive since it acts only in the vicinity of the crossover. Therefore, one may regard

SFD as a "broken extension" of CCC: "broken" because the scale factor inversion is a particular (discrete) Weyl transformation, and "extended" because it isn't valid at a small vicinity of the crossover only. 
The main result presented in this section is that non-trivial SFD-symmetric CCC models $d o$ exist. In other words, the CCC requirements may be implemented within the frameworks of the cyclic SFD expansion/expansion models by self-dual SFD cyclic cosmologies with manifest double $Z_{2} \times Z_{2}$ Weyl symmetry (4.12) (see also eqs. (3.7) and (3.8)). They provide explicit examples of consistent CCC models, whose matter content is given by certain gauged Kähler sigma models (4.10), (4.11).

Within the considered "SFD/CCC context", we can also have SFD symmetric expanding/expanding cosmologies that are not exactly of CCC-type, in which the suppressed rest-mass hypothesis is not satisfied but the continuity of the derivatives of the conformal factors is respected. In other words, the normal vectors $\check{N}_{\mu}=\check{\gamma} \partial_{\mu} \Omega$ and $\hat{N}_{\mu}=\hat{\gamma} \partial_{\mu} \omega$ are continuous on the crossover surfaces $\mathcal{X}=\{\omega=0\}$, but we may include the term linear in $\omega$ in eqs. (4.3) and (4.14). This "weak CCC condition" is compatible, for example, with the $\Lambda$ CDM model

$$
\rho_{\Lambda \mathrm{CDM}}=\rho_{\Lambda}+\frac{\rho_{r}}{a^{4}}+\frac{\rho_{d}}{a^{3}},
$$

which we note that, however, produces a sequence of aeons alternating with universes which contain domain walls instead of dust.

\section{On the thermodynamics of SFD symmetric CCC models}

The interest in studying the thermodynamics of "unconventional cosmologies" such as the pre-big-bang versions of dilaton gravity [15, 37] and the CCC models [17] has to do with the attempts to explain the low entropy of the early universe and/or to reach an alternative description of its inflationary phase. Despite the complexity of the thermodynamical phenomena that occur during the evolution of the observable universe and the lack of complete understanding of the gravitational contributions to the universe entropy, certain model independent estimations of the asymptotic initial and finite values of appropriately defined generalized entropy are available [38, 39] (see also refs. [40, 41]). Their implementation to the case of considered SFD symmetric pre-big-bang and cyclic cosmologies is not straightforward and it turns out to involve several additional requirements and restrictions. The desired thermodynamic features, expected to takes place in the original CCC scenario [17], are known to be quite far from the relatively simple adiabatic thermodynamics of the considered partially self-dual SFD symmetric cyclic models. Nevertheless, the established SFD/CCC relations allows us to gain some important insights on the universal asymptotic (near the crossover) thermodynamical features of the proper CCC models as well.

\subsection{SFD thermodynamics}

The thermal history of each "SFD aeon", filled by a barotropic fluid, ${ }^{16}$ is known to be an adiabatic process (see, e.g. [42]) characterized by

$$
s=\frac{1}{T}(p+\rho)=\frac{S}{V}, \quad E=S T-p V, \quad d s=\frac{d \rho}{T}, \quad \frac{d \rho}{d T}=\frac{1}{T}(p+\rho) \frac{d \rho}{d p},
$$

\footnotetext{
${ }^{16}$ We assume here that $\rho(T)$ and $p(T)$ are functions of the temperature, and that the entropy $S(V, T)$ of the physical volume $V=V_{0} a^{3}$, where $V_{0}$ is a given constant comoving volume, is an extensive quantity with density $s$.
} 
derived from the first law of thermodynamics ${ }^{17} d E=T d S-p d V$, where $E=\rho V$ denotes the internal energy within the physical volume $V=a^{3} V_{0}$. For each fluid with a given EoS $p=w(\rho) \rho$, the above eqs. (5.1) allow to deduce the thermal evolution of $\rho(T), s(T)$ and $a(T)$. One of the most important thermodynamical features of such fluids is that the $2^{\text {nd }}$ TD law, $d S / d t \geq 0$, is trivially satisfied, since the comoving entropy density $\mathcal{S}$ and the entropy of the physical volume $V$ remain constant during the universe evolution:

$$
s a^{3}=\mathcal{S}=\text { const }, \quad S=\mathcal{S} V_{0}=\text { const } .
$$

The SFD transformations of the thermodynamical quantities of the consecutive aeons, say $\hat{S}=\hat{S}(\check{S})$, etc., may be derived by taking into account eqs. (2.11) together with the $E$, $S$ and $T$ relation (5.1), which allows to exclude the pressure $p$, so that

$$
\frac{E_{j}}{a_{j}}=\frac{E_{j+1}}{a_{j+1}}, \quad \frac{1}{a_{j}}\left(S_{j} T_{j}-\frac{2}{3} E_{j}\right)=-\frac{1}{a_{j+1}}\left(S_{j+1} T_{j+1}-\frac{2}{3} E_{j+1}\right) .
$$

We next consider the modified Chaplygin gas models (3.2), whose thermodynamic evolution may be written as

$$
\rho\left(1-\frac{\rho_{\Lambda}^{\delta}}{\rho^{\delta}}\right)^{4-\frac{3}{\delta}}=4 \sigma T^{4}, \quad \mathcal{S} \equiv s a^{3}=\frac{4}{3}\left(4 \sigma \rho_{r}^{3}\right)^{1 / 4}, \quad \infty>\rho \geq \rho_{\Lambda},
$$

obtained from the last of the eqs. (5.1). The constant of integration $4 \sigma$, where $\sigma=\frac{\pi^{2} K_{B}^{4}}{60 \hbar^{3}}$ is the Stephan-Boltzmann constant ( $c=1$ is assumed in all the formulae), has been chosen in such a way that for pure radiation, i.e. for $\rho_{\Lambda} \rightarrow 0$, eq. (5.4) becomes the StephanBoltzmann law, $\rho=4 \sigma T^{4}$. Notice that the above expression for $T(\rho)$ leads to three qualitatively different thermal histories [44]:

- For $0<\delta<\frac{3}{4}$, the temperature $T(a)$ is not a monotonic function, and diverges at both limits $\rho \rightarrow \rho_{\Lambda}$ and $\rho \rightarrow \infty$. Thus the initial big-bang stage and the final (nearly de Sitter) stage are equally hot. The behavior of such models is in disagreement with the expected cooling down of the expanding universe along with the (monotonic) decrease of its curvature. The origin of this rather unphysical behavior turns out to be related to their thermodynamical instability [44], namely the sound velocity square $v_{s}^{2}=\frac{d p}{d \rho}$ for the models with $\delta<\frac{3}{4}$, becomes negative for a certain range of values of the fluid density. ${ }^{18}$

- For all the models with $\delta>\frac{3}{4}$, the temperature is monotonically decreasing for $T \in(\infty, 0)$.

- One of the preferred CCC fluids, with $\delta=3 / 4$ (see end of section 4.4), gives rise to a rather interesting thermal evolution with a non zero finite temperature $T_{\Lambda}=\left(\frac{\rho_{\Lambda}}{4 \sigma}\right)^{1 / 4}=T_{*}$, and with a Stephan-Boltzmann law almost identical to the radiation one.

\footnotetext{
${ }^{17}$ Which is known to be equivalent to the Friedmann equations (1.3), and vice-versa [43].

${ }^{18}$ For a proof of this statement, together with the detailed description of TD features of the considered SFD symmetric cosmologies, see our recent preprint [44].
} 
Hence the modified Chapliging gas models (3.2) are thermodynamically consistent for $\delta$ within the interval $3 / 4 \leq \delta \leq 1$ only. Let us remember that the upper limit is imposed in order to avoid certain "unphysical" behavior of the Ricci curvature $R=2 \rho_{\Lambda}^{\delta}(\rho)^{1-\delta}$, which for $\delta>1$ vanishes at the (still singular) big-bang, and then increases up to a finite value $R_{\Lambda}=2 \rho_{\Lambda}$.

The advantage of SFD symmetric models is that once we know the matter content and its thermodynamical characteristics at a given aeon (say, at arbitrary instant $-\eta_{*}$ ), then the SFD transformations uniquely determine their values at all the other aeons. The temperature and the entropy of a "past aeon" $\hat{\mathcal{A}}$ filled by a modified Chaplygin gas with $\delta \in[3 / 4,1]$ is given by eqs. (5.4). By construction, the "present aeon" $\check{\mathcal{A}}$ has the same matter content (3.2), but with different relative densities $\hat{\rho}_{r} \neq \check{\rho}_{r}$ and $\hat{\rho}_{\Lambda} \neq \check{\rho}_{\Lambda}$, while the physical volume entropies are related by the SFD transformations as $\check{S}=\left(\frac{\check{\rho}_{r}}{\hat{\rho}_{r}}\right)^{\frac{3}{4}} \hat{S}$. Although the direct relation between temperatures, $\check{T}=\check{T}(\hat{T})$, has a rather complicated form, in the limiting cases $\delta=3 / 4$ and $\delta=1$ it simplifies to:

$$
\delta=\frac{3}{4}: \quad \check{\mathcal{T}}^{3}=\frac{\hat{\mathcal{T}}^{3}}{\hat{\mathcal{T}}^{3}-1} ; \quad \delta=1: \quad \check{\mathcal{T}}=\left(\frac{\hat{\rho}_{\Lambda}}{\check{\rho}_{\Lambda}}\right)^{\frac{1}{2}} \frac{1}{\hat{\mathcal{T}}} ; \quad \hat{\mathcal{T}}=\frac{\hat{T}}{\hat{T}_{*}}, \quad \hat{T}_{*}=\left(\hat{\rho}_{\Lambda} \frac{c}{4 \sigma}\right)^{1 / 4}
$$

The above transformations demonstrate that under certain additional restrictions on the EoS of considered Chapligin-like fluids, i.e. for $3 / 4 \leq \delta \leq 1$ only, the UV/IR features of the SFD symmetry takes place. Namely, the late time low temperatures characterizing the accelerated phase of $\breve{\mathcal{A}}$ are mapped to the early time (nearly big-bang) high temperatures in the decelerated phase of $\hat{\mathcal{A}}$. In the case of self-dual models, despite the changes of the energy and the temperature in the vicinity of the crossover, the entropy of the considered physical volumes remains unchanged, i.e. we have $\hat{S}=\check{S}$ due to $\hat{\rho}_{r}=\check{\rho}_{r}$. We should mention that the TD's of a given physical volume is "causally consistent" when considered far enough from the crossover. It becomes however problematic in the vicinity of the crossover (when it is approached from the final de Sitter region $\check{a} \rightarrow \infty$ of the past aeon), since now a part of the physical volume becomes unobservable - causally disconnected due to the fact that its size overpasses the event horizon. A more consistent description of the thermodynamics of the observable universe can be reached by considering the entropy of the matter within the event or apparent horizons.

\subsection{SFD for horizons thermodynamics}

The apparent horizon is a marginally anti-trapped spherical surface centered at the observer position, its physical radius $\Upsilon_{A}=1 /|H|$ (for $K=0$ ), coincides with the Hubble radius where the velocity of space-time expansion becomes grater than the speed of light. Because of its (local) causal nature, one may assign a kind of Bekenstein-Hawking thermodynamics to apparent horizons, with entropy and temperature ${ }^{19}[45,46]$

$$
T_{A}=\frac{1}{2 \pi \Upsilon_{A}}, \quad S_{A}=\frac{1}{4 G}\left(4 \pi \Upsilon_{A}^{2}\right)=\frac{8 \pi^{2}}{H^{2}},
$$

\footnotetext{
${ }^{19}$ Recall that we use units in which $G=1 / 8 \pi$.
} 
such that the Clausius relation $d Q=T_{A} d S_{A}$ indeed holds as a consequence of the Friedmann equations (1.3) and vice-versa [45]. Due to the relations $H=\sqrt{\rho / 3}=1 / \Upsilon_{A}$, the SFD transformations of the apparent horizon TD quantities can be easily obtained from eqs. (2.3):

$$
\hat{\Upsilon}_{A} / \hat{a}=\check{\Upsilon}_{A} / \check{a} \quad\left(\text { that is } \quad \hat{r}_{A}=\check{r}_{A}\right), \quad \text { and } \quad \hat{a} \hat{T}_{A}=\check{a}_{\bar{T}}, \quad \hat{S}_{A} / \hat{a}^{2}=\check{S}_{A} / \check{a}^{2},
$$

where $r_{A}$ is the apparent horizon's comoving radius.

In the case of partially self-dual SFD symmetric CCC models based on modified Chaplygin gas (3.2), the entropy dependence of the scale factor reads: $a^{-4 \delta}=\frac{\rho_{\Lambda}^{\delta}}{\rho_{r}^{\delta}}\left(\frac{S_{\Lambda}^{\delta}}{S_{A}^{\delta}}-1\right)$. Then, given the past aeon apparent horizon entropy $\hat{S}_{A}$, the SFD transformation (5.7) determines the entropy $\check{S}_{A}$ at the present aeon as

$$
\check{S}_{A}=\frac{\check{S}_{\Lambda}}{\hat{S}_{\Lambda}}\left(\hat{S}_{\Lambda}^{\delta}-\hat{S}_{A}^{\delta}\right)^{\frac{1}{\delta}}, \quad S_{\Lambda} \equiv \frac{24 \pi^{2}}{\rho_{\Lambda}},
$$

with $S_{\Lambda}$ denoting the maximal value of the apparent horizon entropy in the asymptotic future, defined as one forth of the event horizon area at the crossover. It coincides in this limit with the event horizon entropy (and indeed with the actual space-time entropy) of de Sitter space.

The entropy of the observed universe includes, of course, the contribution of the entropy $S_{f}$ of the matter within the apparent horizon. So we have the total (generalized) entropy

$$
\begin{aligned}
S_{t} & =S_{f}+S_{A}=s \times V_{A}+2 \pi A_{A}, \\
V_{A} & =\frac{4}{3} \pi H^{-3}, \quad A_{A}=4 \pi H^{-2}, \quad S_{f}=\mathcal{S} \frac{4 \pi}{3} r_{A}^{3}=\frac{4 \pi}{3} \frac{\mathcal{S}}{(a H)^{3}}, \quad r_{A}=1 /(a H),
\end{aligned}
$$

where $V_{A}$ e $A_{A}$ are the physical volume and area of the apparent horizon. The transformation of this total entropy between aeons is non-homogeneous and given by

$$
\check{S}_{t}=\left(\frac{\hat{\rho}_{\Lambda}}{\check{\rho}_{\Lambda}}\right)^{\frac{3}{4}} \hat{S}_{f}+\left(\frac{\hat{\rho}_{\Lambda}}{\check{\rho}_{\Lambda}}\right) \hat{S}_{A}\left(\frac{\hat{S}_{\Lambda}^{\delta}}{\hat{S}_{A}^{\delta}}-1\right)^{\frac{1}{\delta}},
$$

which in the self-dual case, when $\hat{\rho}_{\Lambda}=\check{\rho}_{\Lambda}$, simplifies to

$$
\check{S}_{t}=\hat{S}_{t}+\hat{S}_{A}\left[\left(\frac{\hat{S}_{\Lambda}^{\delta}}{\hat{S}_{A}^{\delta}}-1\right)^{\frac{1}{\delta}}-1\right] .
$$

The proof is a straightforward consequence of the $S_{A}$ transformations (5.8) and of the fluid entropy SFD law $\hat{S}_{f}=\left(\frac{\check{\rho}_{\Lambda}}{\hat{\rho}_{\Lambda}}\right)^{\frac{3}{4}} \check{S}_{f}$.

Differently from the constant entropy of an arbitrary physical volume (see eq. ( 5.2)), the matter fluid entropy $S_{f}$ is now time dependent and it turns out to satisfy the $2^{\text {nd }}$ law in a decelerated matter/radiation phase only, i.e. when $q(a)=\frac{1}{2}\left(1+3 \frac{p}{\rho}\right) \geq 0$, since

$$
\dot{S}_{f}=\frac{2 \pi}{3} \frac{\mathcal{S} a}{(a H)^{4}}(\rho+3 p) \geq 0
$$


The origin of the violation of the $2^{\text {nd }}$ law for $S_{f}$ during the accelerated phase of the universe (i.e for $q<0$ ) is related to the decreasing of the comoving volume $V_{A}$ of the apparent horizon in this case.

The problem we are obliged to face up here is about the conditions that might ensure the validity of the generalized $2^{\text {nd }}$ law, $\dot{S}_{t} \geq 0$, during the evolution at a given aeon. For the modified Chaplygin gas (3.2), we have

$$
\frac{d S_{t}}{d a}=\frac{96 \pi^{2} \rho_{r}^{\delta}}{a^{4 \delta+1}} \frac{1}{\left(\rho_{\Lambda}^{\delta}+\rho_{r}^{\delta} a^{-4 \delta}\right)^{\frac{1}{\delta}+1}}\left[1+\frac{\sqrt{3} \mathcal{S}}{8 \pi \rho_{r}^{\delta} a^{3}} \frac{\left(\rho_{r}^{\delta}-\rho_{\Lambda}^{\delta} a^{4 \delta}\right)}{\left(\rho_{\Lambda}^{\delta}+\rho_{r}^{\delta} a^{-4 \delta}\right)^{\frac{1}{2 \delta}}}\right] \geq 0
$$

where we have used $H=\sqrt{\frac{\rho}{3}}$ with $\rho$ given by eq. (3.2). We must consider the validity of the above inequality only within the physical interval where $3 / 4 \leq \delta \leq 1$. The result may be summarized in the following statement:

The $2^{\text {nd }}$ law holds during the entire evolution of the observable universe, i.e. for all $\eta \in\left[0, \eta_{f}\right]$, only for the $\delta=3 / 4$ model, and provided its vacuum density satisfies a distinct upper bound

$$
\rho_{\Lambda}^{1 / 4} \leq \frac{2 \pi \sqrt{3}}{(4 \sigma)^{1 / 4}}
$$

( $c=1=\hbar ; \sigma$ the Steffan-Boltzmann constant.) This restriction on the maximal allowed value of the cosmological constant introduces a lower bound, $S_{\Lambda} \geq \frac{2 \sigma}{3 \pi^{2}}$, on the maximal entropy of the universe filled with the $\delta=3 / 4$ modified Chaplygin gas.

Its proof is presented in appendix B. This result establishes the TD consistency of a distinct cosmological model (3.2) within the framework of one particular thermodynamical description of the observable universe involving apparent horizons [45, 46]. It also illustrates the difficulties inherent to the definition of a generalized entropy in cosmology. When the universe enters an accelerated phase, the comoving radius of the apparent horizon shrinks, as noted in eq. (5.12), and in fact it vanishes as one asymptotes towards de Sitter space-time. Therefore the total (adiabatic) entropy of the fluid inside it naturally decreases, as the sphere inside this horizon becomes empty. If one simply adds this decreasing entropy of the fluid with the Bekenstein-Hawking entropy of the horizon itself, the resulting "total entropy" $S_{t}$ will only obey the $2^{\text {nd }}$ Law for very specific cases in which the increasing (physical) area dominates the decreasing (comoving) volume. Eq. (5.13) shows that, although relatively "rare", such universes do exist. It is more natural however to seek for another definition of the generalized entropy that ensure the validity of the $2^{\text {nd }}$ Law for the observable universe filled by physically relevant fluid densities, say for the $\Lambda$ CDM model at least. A different approach to the total entropy, based on a new area-theorem [41], was recently proposed in ref. [40]. Their assertion is that the generalized entropy - matter entropy inside a volume, plus one quarter of the corresponding area - always increases monotonically along a specifically constructed hypersurface called the holographic Q-screen, which (generally) differs slightly from the apparent horizon. 


\subsection{Gauss-Bonnet extension and crossover entropy}

Once we choose to work with the generalized entropy (5.9), the problem arises of whether one can extend the above results to the cyclic SFD symmetric CCC models - what are the eventual additional conditions to be imposed on the behavior of the entropy $S_{t}$ in the vicinity of the crossover? Should we assign a certain amount of entropy to each crossover surface $\mathcal{X}_{j}$ ? Must the generalized entropy also increase from one aeon to the next one during the cyclic evolution of the universe?

The first problem to be solved, however, concerns the zero value of the total entropy at the big-bang in a radiation dominated early universe: for $a \rightarrow 0$,

$$
S_{t} \approx \frac{4 \pi \sqrt{3}}{\rho_{r}^{3}} \mathcal{S} a^{3}+\frac{24 \pi^{2}}{\rho_{r}} a^{2} \rightarrow 0 .
$$

Such an "unphysical" zero entropy initial state of the universe evolution is due to the fact that the radius of the apparent horizon vanishes at the big-bang. Therefore, on the vicinity of the crossover, its area $A_{A}=4 \pi H^{-2}$ becomes smaller than the Planck area, ${ }^{20}$ i.e. $A_{A}<A_{p l}=4 \pi l_{p l}^{2}=16 \pi^{2} G$, which is beyond of the scale of validity of classical Einstein gravity. One solution to this problem consists in the modification of the apparent horizon's entropy by an additive constant: $\bar{S}_{A}=S_{A}+S_{0}$. The extra entropy $S_{0}$ could be related to quantum corrections of the Einstein action, or else to scale (or conformal) invariant terms added to it in order to describe the degrees of freedom prescribed to the conformal crossover. Gauss-Bonnet (GB) Gravity, with action ${ }^{21}$

$$
S_{\mathrm{GB}}=\int \frac{\sqrt{-g} d^{4} x}{16 \pi G}\left\{R-\lambda L^{2}\left[2 R^{2}-8 R_{\alpha \beta} R^{\alpha \beta}+2 R_{\alpha \beta \mu \nu} R^{\alpha \beta \mu \nu}\right]+\mathcal{L}_{\text {matter }}\right\},
$$

represents a quite reasonable example for such an improvement of Einstein Gravity that provides an explicit form of the desirable new constant term (for $\lambda<0$ ) in the apparent horizon entropy [45, 47-49]:

$$
\bar{S}_{A}=8 \pi^{2} \frac{1}{H^{2}}\left(1-2 \lambda L^{2} H^{2}\right)=S_{A}+S_{0}, \quad S_{0}=8 \pi^{2}|\lambda| \frac{L^{2}}{l_{p l}^{2}} .
$$

An important feature of the GB term is that it is scale invariant, and being also a topological invariant it does not contribute to the equations of motion. Thus the Friedmann eqs. (1.3) keep their form and, as a consequence, their SFD properties remain unchanged. Therefore, all the SFD constructions and the CCC and TD restrictions on the matter content we have derived (for Einstein Gravity) in sections 3, 4 and 5 are still valid for the GB gravity as well.

With this "GB renormalization", the total entropy becomes

$$
S_{t}=S_{f}+S_{A}+S_{0}
$$

\footnotetext{
${ }^{20}$ In the units used in this paper, with $8 \pi G=1$, we have $A_{p l}=2 \pi$, i.e. $l_{p l}^{2}=1 / 2$.

${ }^{21}$ Here $\lambda$ is a new dimensionless "gravitational coupling" constant, while $L$ is a new length scale which can be chosen as $L=l_{p l}$ by an appropriate redefinition of $\lambda$; the cosmological constant $\rho_{\Lambda}=\Lambda>0$ is included in the potential $V(\sigma)$ of the matter Lagrangian $\mathcal{L}_{\text {matter }}$.
} 
The addition of a constant GB entropy $S_{0}$ slightly modifies the transformation law (5.10). If we do not impose any additional requirements relating $\hat{S}_{0}$ and $\check{S}_{0}$, the SFD transformation for the GB improved total entropy (5.18) takes the from

$$
\check{S}_{t}=\left(\frac{\hat{\rho}_{\Lambda}}{\check{\rho}_{\Lambda}}\right)^{\frac{3}{4}} \hat{S}_{f}+\left(\frac{\hat{\rho}_{\Lambda}}{\check{\rho}_{\Lambda}}\right) \hat{S}_{A}\left(\frac{\hat{S}_{\Lambda}^{\delta}}{\hat{S}_{A}^{\delta}}-1\right)^{\frac{1}{\delta}}+\check{S}_{0}
$$

or, in the self-dual case, $\check{S}_{t}=\hat{S}_{t}+\hat{S}_{A}\left[\left(\hat{S}_{\Lambda}^{\delta}-1\right)^{\frac{1}{\delta}}-1\right]+\check{S}_{0}-\hat{S}_{0}$. The main importance of $S_{0}$ resides in that we may regard it as an entropy of the crossover, related to the specific conformal field theory valid at the immediate vicinity of $\mathcal{X}$. On the "past" side of $\mathcal{X}$, the pre-big-bang entropy is given by $\hat{S}_{t}=\hat{S}_{\Lambda}$ (cf. eq. (5.8)). If there was no GB entropy, on the "future" side of $\mathcal{X}$, we would have (the problematic) $\check{S}_{t}=0$. Thus the conformal theory on the crossover would be responsible for a "complete loss of memory" between two consecutive aeons. The effect of the presence of $\check{S}_{0}$ is, therefore, that it allows us to keep a "partial persistency of the memory" across the crossovers.

Let us look in detail at what happens to entropy when one passes between consecutive aeons in a cyclic sequence, say $\mathcal{A}_{0}, \mathcal{A}_{1}$ and $\mathcal{A}_{2}=\mathcal{A}_{0}$. We further denote $\mathcal{A}_{0}=\hat{\mathcal{A}}$ and $\mathcal{A}_{1}=\check{\mathcal{A}}$. There are two crossovers $\mathcal{X}_{0}$ and $\mathcal{X}_{1}$, cf. figure 3 . Each has a corresponding GB entropy and we shall assume that the GB scales $\hat{L}$ and $\check{L}$ are different, with $\hat{L} \leq \check{L}$ (such that $\hat{S}_{0} \leq \check{S}_{0}$ ), while the GB coupling $\lambda$ remains unchanged. The total entropies behave, near the first crossover $\mathcal{X}_{0}$, as $\left.\hat{S}_{t}\right|_{\hat{a} \rightarrow \infty}=\hat{S}_{0}+\hat{S}_{\Lambda}$ and $\left.\check{S}_{t}\right|_{a \rightarrow 0}=\check{S}_{0}$. Therefore, the entropy losses on each crossover are given by:

$$
\begin{aligned}
& \Delta S_{\mathcal{X}_{0}}=\left(\hat{S}_{0}+\hat{S}_{\Lambda}\right)-\check{S}_{0}=\frac{4 \pi^{2}}{l_{p l}^{2}}\left[2|\lambda|\left(\hat{L}^{2}-\check{L}^{2}\right)+\hat{L}_{d S}^{2}\right], \\
& \Delta S_{\mathcal{X}_{1}}=\left(\check{S}_{0}+\check{S}_{\Lambda}\right)-\hat{S}_{0}=\frac{4 \pi^{2}}{l_{p l}^{2}}\left[2|\lambda|\left(\check{L}^{2}-\hat{L}^{2}\right)+\check{L}_{d S}^{2}\right],
\end{aligned}
$$

where we have used the de Sitter radii $L_{d S}$, with $\check{\rho}_{\Lambda}=3 / \check{L}_{d S}^{2}$ and $\hat{\rho}_{\Lambda}=3 / \hat{L}_{d S}^{2}$. Note that there is one special choice for the GB coupling,

$$
|\lambda|=\frac{\hat{L}_{d S}^{2}}{2\left(\check{L}^{2}-\hat{L}^{2}\right)},
$$

that ensures the continuity of the entropy on $\mathcal{X}_{0}$ (and on all the equivalent crossovers $\left.\mathcal{X}_{0}=\mathcal{X}_{2}=\mathcal{X}_{4}=\cdots\right)$, viz. $\Delta S_{\mathcal{X}_{0}}=0$. But it is impossible to make it continuous on the next (or on the previous) crossover as well, since now we have $\Delta S_{\mathcal{X}_{1}}=\frac{4 \pi^{2}}{l_{p l}^{2}}\left(\check{L}_{d S}^{2}+\hat{L}_{d S}^{2}\right)$. In the self-dual case, i.e. for $\check{\rho}_{\Lambda}=\hat{\rho}_{\Lambda}$ and for equal GB scales $\check{L}=\hat{L}$, the losses of entropy at the different crossovers are identical: $\Delta S_{\mathcal{X}_{0}}=\Delta S_{\mathcal{X}_{1}}=\cdots=S_{\Lambda}$, as well as its evolution inside all the aeons - it always increases from $S_{0}$ to $S_{0}+S_{\Lambda}$.

An important ingredient defining the described entropy evolution is the specific choice of GB entropy contributions at different aeons. Within the framework of the SFD symmetric cosmologies, one can also consider another semi-infinite SFD extension, representing a chain of aeons $\mathcal{A}_{j}$ with different GB scales $L_{j}^{2}=(j+1) l_{p l}^{2}$ at different aeons. This chain 
begins with a "primordial crossover" $\mathcal{X}_{0}$ of minimal entropy $S_{0}=4 \pi^{2}|\lambda|$, and the total entropy of a future aeon $\mathcal{A}_{j}$ is given by

$$
S_{t}(j)=S_{f}(j)+S_{\Lambda}(j)+(j+1) S_{0}
$$

where $S_{0}(j)=(j+1) S_{0}$ is the GB contribution. The effect of this particular form of the GB entropies $S_{0}(j)$ may be also interpreted as an extension of the SFD entropy definition, that permits an accumulation of the "primordial GB entropy" $S_{0}=4 \pi^{2}|\lambda|$ along the consecutive self-dual aeons. We may depict the evolution as follows:

$$
\mathcal{X}_{0}\left\|S_{0} \hookrightarrow S_{0}+S_{\Lambda}\right\| \mathcal{X}_{1}\left\|2 S_{0} \hookrightarrow 2 S_{0}+S_{\Lambda}\right\| \mathcal{X}_{2}\left\|3 S_{0} \hookrightarrow 3 S_{0}+S_{\Lambda}\right\| \mathcal{X}_{3} \| \cdots
$$

It is clear that all the entropy losses $\Delta S_{\mathcal{X}_{j}}$ are now identical by construction,

$$
\Delta S_{\mathcal{X}_{j}}=S_{\Lambda}-S_{0}=\frac{4 \pi^{2}}{l_{p l}^{2}}\left(L_{d S}^{2}-2|\lambda| l_{p l}^{2}\right),
$$

and we have an equal partial loss of entropy at each crossover. Therefore, we may reach the continuity of the entropy on all the crossovers by choosing $|\lambda|=L_{d S}^{2} / 2 l_{p l}^{2}$. Such a choice ensures not only the continuity, but also the "eternal" increasing of the entropy (say for $\delta=3 / 4$ model (3.2)) considered for the entire infinite chain of aeons.

The above two examples of different choices of the values of the GB parameters demonstrate how the additional requirement on the form of the GB term at different aeons can give rise to qualitatively different behaviors of the entropy evolution in the SFD symmetric expansion/expansion models. Hence a more complete investigation of the crossover features is needed in order to select one among the many allowed entropy evolutions of such CCC-like models.

\section{Concluding remarks}

Veneziano's idea [14] of using scale factor inversion as a symmetry principle for constructing pre-big-bang extensions of the FRW solutions of Dilaton Gravity has been shown to have a successful implementation for pre-big-bang and cyclic cosmologies also in Einstein Gravity (and its Gauss-Bonnet extension), when the conformal time SFD transformations [23] are imposed as a symmetry of these cosmological models. The possibility of combining the scale factor inversions with conformal time translations (2.5) or with reflections (2.3) gives rise to two distinct pre-big-bang evolutions - the contraction/expansion and the expansion/expansion ones.

In the present paper we have chosen to study in more detail the geometric and thermodynamical consistency of SFD symmetric models of the expansion/expansion type and their application in the construction of relevant examples of Penrose's Conformal Cyclic Cosmologies [17]. The fact that the employment of the scale factor duality ideas to CCC models is an unexplored area that deserves to be investigated is only one of our motivations. The results presented in sections 4 and 5 demonstrate the power of the SFD requirement - when applied to CCC, it allows us to describe all the physical quantities characterizing 
the present aeon in terms of the ones of the past aeon and vice-versa. It also imposes certain restrictions on the evolution of the total entropy of the observable universe in the SFD dual aeons (and on its behavior on the crossover), compatible with the generalized TD second law. Finally, the SFD symmetry picks out a family of relevant examples of self-dual cosmologies, whose matter content coincides with the $\mathrm{S} U(1,1) / \mathrm{U}(1)$ gauged Kähler sigma models with the specific self-interaction (4.10).

Our concluding remarks concern some of the virtues and the drawbacks of these prebig-bang and cyclic cosmological models, in search for convincing arguments about the relevance of conformal time SFD as an asymptotic UV/IR symmetry of the universe evolution. The comparison of the main features of the CCC-like expansion/expansion models with their contraction/expansion relatives aims to clarify the benefits of the employment of the former as unconventional cosmological models. Within the description of the physically consistent realizations of the restrictions imposed by scale factor duality, we will briefly discuss some consequences of the SFD requirement on the properties of the adiabatic fluctuations around the SFD symmetric FRW backgrounds.

\subsection{More on contraction/expansion SFD models}

As we have shown in sections 2 and 3, for each choice of dual (or self-dual) pairs of matter fluids, one finds two kinds of SFD symmetric FRW solutions, corresponding to the two realizations (2.3) and (2.5) of the conformal time scale factor duality (1.2). The different nature of the pre-big-bang phases of the expanding/expanding (E/E) and contracting/expanding $(\mathrm{C} / \mathrm{E})$ solutions lead to very different transitions between the corresponding dual phases: (i) the big-crunch/big-bang singularity at $\hat{a}=0=\check{a}$, versus (ii) the conformal crossover, where one identifies $\check{a}=\infty$ with $\hat{a}=0$. Let us recall the action of the SFD transformations used to "glue" such pairs of dual solutions: in the case of C/E models, they map the initial pre-big-bang accelerated contraction phase into the initial decelerated expansion phase of the next post-big-bang epoch, while for the E/E models, the initial decelerated expansion phase of the past aeon is the SFD "image" of the final accelerated expanding phase in the future aeon and vice-versa.

The SFD symmetric C/E models, studied in sections 2 and 3, provide examples of contraction/expansion FRW backgrounds with a reasonable matter content satisfying the weak energy condition. The self-duality of the matter potential $V(\sigma)(3.15)$, defining cosmological models with modified Chaplygin gas EoS (3.2), is the main responsible for the continuity of the scale factor $a(\eta)$, of the scalar field $\sigma(\eta)$ and of their derivatives at the big-crunch/big-bang singularity at $\eta=0$. Their partially self-dual counterparts offer an interesting $\mathrm{C} / \mathrm{E}$ cosmological scenario with initial and final "de Sitter states" of different cosmological constants $\check{\rho}_{\Lambda} \neq \hat{\rho}_{\Lambda}$. However, the derivatives of the scale factors at the singularity now present finite jumps due to the different relative radiation densities $\check{\rho}_{r} \neq \hat{\rho}_{r}$. It is worthwhile to mention that these features are also shared by the $\mathrm{C} / \mathrm{E}$ cosmology involving $\Lambda$ CDM model and its SFD dual (1.4).

The cosmological feasibility of the contraction/expansion models depends on the eventual instabilities and ghosts problems that might be caused by the requirement of scale factor duality as an asymptotic symmetry of the universe evolution. The appearance of 
such inconsistencies (and the ways they might be avoided) are mainly related to the properties of the adiabatic fluctuations around the contraction phase of the SFD symmetric backgrounds. The similarity of certain features of our C/E models with those of the Prebig-bang Scenario, and the Ekpyrotic and Cyclic cosmologies, suggests that we can make the discussion of their flaws shorter by borrowing and readapting some of the arguments from the extensive critical analysis of those models presented in refs. [18, 19, 37, 50-52]. One should stand out a few of the problems to be faced - the chaotic anisotropic BelinskiiKhalatnikov-Lifshitz-like (BKL) singularities [53, 54]; the gradient and thermodynamical instabilities and the absence of ghosts, phantoms and other WEC breaking inconsistencies.

The cosmological models whose EoS parameter is bounded as $w<1$ in the contraction phase are known to develop chaotic BKL "mixmaster" behavior near the singularity. Therefore all the considered SFD dual and self-dual C/E models indeed suffer from BKL inconsistency, ${ }^{22}$ due to the "SFD restrictions" $-1 \leq w(\rho) \leq 1 / 3$ imposed on the weak energy conditions.

In the ekpyrotic/cyclic models [18] the BKL chaotic singularity is avoided via a mechanism that makes the (effective) EoS parameter $w \gg 1$ as the universe collapses. A new ekpyrotic (with low density and low curvature) slow contraction phase is created by a specific scalar field potential with a negative valley, or else by considering a "ghost condensate" matter content $[55,56]$. Although they are not free from certain "ekpyrotic ghost" inconsistencies [51], they turn out to provide a reasonable contracting pre-big-bang alternative of the standard inflation scenario. It is then natural to try to incorporate the particular features of these models within SFD symmetric C/E cosmologies, in order to make them free of BKL singularities. However, the SFD dual of an ekpyrotic phase with $\hat{w} \gg 1$ must have $\check{w}=-\hat{w}-\frac{2}{3} \ll-1$. So it turns out that the WEC is then strongly violated in the expanding post-big-bang phase of these ekpyrotic SFD models and, as a consequence, they are subject to dangerous "phantom" [51, 57] and gradient instabilities [52]. We have to also notice that the SFD symmetric ekpyrotic cosmologies do not reproduce one of the basic features of the original ekpyrotic/cyclic models, namely that their post-big-bang phase should be given by a standard $\Lambda$ CDM model.

In brief, the problems of SFD symmetric $\mathrm{C} / \mathrm{E}$ models demonstrates that their ekpyrotic (BKL free) improvements also fail to represent consistent cosmologies, due to the presence of ghosts and gradient instabilities induced by the SFD requirement.

\subsection{On the scale factor duality of adiabatic fluctuations}

Fluctuations around the homogeneous and isotropic backgrounds are expected to break down the scale factor duality, mainly due to the extra scales involved in the observed nonhomogeneous and anisotropic evolution of the universe. In the case of SFD symmetric $\mathrm{C} / \mathrm{E}$ models, the construction of a smooth bounce (that should replace the singularity) also necessarily introduces certain higher derivative "quantum corrections" to Einstein gravity (i.e. powers of the curvature invariants), that are known to be incompatible with

\footnotetext{
${ }^{22}$ We have to remind that the SFD models with EoS (3.2) for $\delta<0$ are in fact non-singular but represent certain TD consistency problems [44] and due to $v_{s}^{2}<0$ they might develop gradient instabilities as well.
} 
the scale factor duality. Such a problem is, however, absent in the considered CCC-like models, due to the special features of the conformal crossover as the transition between two consecutive aeons. The cosmological applications of such models and the use of their late time accelerated phase as a "pre-big-bang inflationary" phase crucially depends on the existence of a consistent mechanism for the transmission of the fluctuations through the conformal crossover.

The well known S-duality extension [58] of the original SFD symmetry ${ }^{23}$ of Dilaton Gravity provides an indication about the possible realizations of the SFD transformations of the tensor modes (i.e. of helicities \pm 2 ) of metric fluctuations $g_{ \pm}\left(\eta, x_{i}\right) \approx a^{2}(\eta)\left(1+h_{ \pm}\left(\eta, x_{i}\right)\right.$ ) that keep invariant their energy density. By considering the second order terms in the expansion of the Einstein Gravity action, one can derive the contribution of the tensor fluctuations to the corresponding Hamiltonian density:

$$
\mathcal{H}=\frac{1}{2} \int d^{3} x\left[\frac{\Pi^{2}}{a^{2}(\eta)}+a^{2}(\eta)(\nabla h)^{2}\right]=\frac{1}{2} \int d^{3} k\left[\frac{\left|\Pi_{k}\right|^{2}}{a^{2}(\eta)}+k^{2} a^{2}(\eta)\left|h_{k}\right|^{2}\right],
$$

where $h$ is either one of the transverse traceless modes $h_{ \pm}$(i.e. $h_{j}^{j}=0$ and $\nabla^{j} h_{j i}=0$ ) of the gravitational wave; $\Pi=a^{2} h^{\prime}$ their canonically conjugate momenta; $h_{k}, \Pi_{k}$ the corresponding Fourier modes (with wavenumber $k$ ) and the abreviations $\left|h_{k}\right|^{2}=h_{k} h_{-k}$ and $k^{2}=|k|^{2}=k_{i} k_{i}$ have been assumed. It may be easily verified that the Hamiltonian and, consequently, the equations of motion for the fluctuations $h_{k}$ and for their momenta $\Pi_{k}$ are invariant under the transformations

$$
a \mapsto \tilde{a}(\tilde{\eta})=c_{0}^{2} / a(\eta), \quad h_{k} \mapsto \tilde{h}_{k}(\tilde{\eta})=-\frac{1}{c_{0}^{2} k} \Pi_{k}(\eta), \quad \Pi_{k} \mapsto \tilde{\Pi}_{k}(\tilde{\eta})=c_{0}^{2} k h_{k}(\eta),
$$

consisting of the scale factor inversion (1.2a) along with an appropriate exchange of the fields with their momenta. Such an enhancement of the SFD symmetry by a particular canonical transformation allows to completely determine the tensor modes $\check{h}_{k}(\eta)$ of the metric fluctuations in the early time (radiation/matter dominated) post-big-bang phase in terms of their SFD dual (late-time) accelerated pre-big-bang counterparts $\hat{h}_{k}(-\eta)$,

$$
\check{h}_{k}(\eta)=\frac{1}{c_{0}^{2} k} \hat{a}^{2}(-\eta) \hat{h}_{k}^{\prime}(-\eta), \quad 0 \leq \eta \leq \eta_{f} .
$$

It remains however to establish the prescription of how to follow these metric perturbations through the crossover surface $\mathcal{X}$, i.e. to define appropriate matching rules for the solutions $\check{h}_{k}$ and $\check{h}_{k}$ at $\eta=0$. The SFD symmetry requirement (6.3), together with the natural condition for (asymptotic) vanishing of the fluctuations $\bar{h}(\eta)=a(\eta) h_{k}(\eta)$ at the homogeneous and isotropic initial/final stages of the universe evolution, can be satisfied by imposing $\hat{\bar{h}}_{k}(0)=0=\check{\bar{h}}_{k}(0)$ and permitting a finite jump $\Delta \bar{h}_{k}=\hat{\bar{h}}_{k}^{\prime}(0)-\check{\bar{h}}_{k}^{\prime}(0)$ of their derivatives. It is worthwhile to mention that in the case of $\delta=1 / 2$ self-dual model, where the fluctuation equations are exactly solvable, the above "crossover conditions" allow to

\footnotetext{
${ }^{23}$ See section 2 of ref. [23] for a comparison of its similarities and differences with the considered conformal time SFD (1.2).
} 
determine the (k-dependent) coefficients of the solutions for $\hat{h}_{k}(-\eta), \check{h}_{k}(\eta)$ and the exact form $k_{n}^{2}$ of their discrete spectrum as well.

The scalar modes $\Psi\left(\eta, x_{i}\right)$ of the metric perturbations in longitudinal gauge (and in the absence of anisotropic stresses), $d s^{2}=a^{2}(\eta)\left[-(1+2 \Psi) d \eta^{2}+(1-2 \Psi) \delta_{i j} d x^{i} d x^{j}\right]$ obey the equations

$$
u_{k}^{\prime \prime}+\left[k^{2}-\frac{z^{\prime \prime}}{z}\right] u_{k}=0, \quad u_{k}=z \Psi_{k}, \quad z=a^{2} \frac{\sigma^{\prime}}{a^{\prime}}
$$

that are similar to the ones for the tensor modes, when written for $\bar{h}_{k}=a h_{k}$. However the difference between their Barden potentials $\left(V_{\bar{h}}=\frac{a^{\prime \prime}}{a}\right.$ and $\left.V_{u}=\frac{z^{\prime \prime}}{z}\right)$ as well as the different SFD transformation properties of the variables $a$ and $z$ do not permit ${ }^{24}$ to extend the background scale factor duality (1.2) as we have done for the corresponding tensor modes. Although now one cannot use the SFD symmetry in order to define the post-big-bang scalar fluctuations in terms of the corresponding pre-big-bang ones, an important question to be addressed concerns the consequences of their SFD dual matter content (and of the specific SFD symmetric backgrounds) on the properties of fluctuations and of their spectra. The background SFD symmetry in fact completely determine the potentials $\hat{V}_{\hat{u}}(\hat{z}) \neq \check{V}_{\breve{u}}(\check{z})$ of the scalar mode fluctuation equations at two consecutive aeons. For example, the near crossover approximation of the potentials $V_{u}=\frac{z^{\prime \prime}}{z}$ corresponding to the self-dual SFD models (3.2) takes a rather familiar (but different) form at the both sides of the crossover surface $\mathcal{X}=[\{\hat{a} \rightarrow \infty\} \sim\{\check{a} \rightarrow 0\}]$ :

$$
\hat{u}_{k}^{\prime \prime}+\left[k^{2}-\frac{\nu^{2}-\frac{1}{4}}{\eta^{2}}\right] \hat{u}_{k}=0, \quad \text { for } \quad \hat{\eta} \rightarrow 0^{-}, \quad \text { and } \quad \check{u}_{k}^{\prime \prime}+k^{2} \check{u}_{k}=0, \quad \text { for } \quad \check{\eta} \rightarrow 0^{+}
$$

(with $\left.\nu=2 \delta-\frac{3}{2}\right)$, which are derived by using a simplified $z=-2\left(\frac{\rho_{r}}{3}\right)^{\frac{\delta}{2}} a^{1-\delta}(a H)^{-\delta}$ form in the evaluation of the limits $\hat{a} \rightarrow \frac{1}{\eta}$ and $\check{a} \rightarrow \eta$. Their solutions are indeed well known and it seems quite reasonable to impose for $\hat{u}_{k}$ and $\check{u}_{k}$ a crossover prescription similar to the one used for the tensor modes. Although such a choice provides consistent answers for $\delta=1$ and $\delta=1 / 2$ self-dual models, the derivation of the generic form of the scalar modes crossover matching conditions requires further investigations.

The proper structure of all the SFD symmetric CCC models opens a room for certain speculations about the eventual double role of the accelerated asymptotically $d S_{4}$ phase at the vicinity of crossover. One may try to derive the restrictions under which it can serve as a pre-big-bang inflationary phase of superluminal expansion and cooling up to almost zero temperature, and even more - that the conformal crossover could play the role of a kind of reheating transition to the radiation dominated (high temperature) post-big-bang phase. The solutions of the horizon and flatness problems in these considered CCC models are quite similar to those offered by the well known dilaton gravity pre-big-bang models and some of the cyclic models as well. The question concerning the existence (or not) of conditions favorable for the complete realization of such pre-big-bang inflationary scenario is, however, out of the scope of the present paper.

\footnotetext{
${ }^{24}$ With an exception of the particular cases, when $z \sim a$, as for example for perfect fluids.
} 


\subsection{Towards the conformal crossover holography}

As we have shown in section 4.1, the origin of the asymptotic SFD symmetry of CCC models is closely related to the "reciprocal hypothesis" and to the conformal crossover, i.e. the existence of conformal (Weyl) equivalence class of metrics defining the transition between the future infinity of one aeon to the big-bang singularity of the next one. We have deduced a set of SFD requirements concerning the conformal properties of the crossover and also a particular mechanism for the explicit breaking of this crossover's conformal symmetry, namely by adding certain relevant operators preserving unbroken the subgroup of scale factor inversions. We consider these results as a step towards the realization of the $4 \mathrm{D}$ conformal field theories ${ }^{25}$ describing these transition regions. The similarity of the primordial conformal crossover, introduced in section 5.3, to the original ideas of conformal and pseudo-conformal initial stage of the universe evolution [4, 5, 59-61], suggests that they could be realized within the frameworks of the SFD symmetric CCC models as well.

Another conformal byproduct of our investigation of the SFD/CCC correspondence are the following two indications of how one can implement the $d S_{4} / C F T_{3}$ holographic meth$\operatorname{ods}^{26}[65,66]$ for the description of the conformal crossovers in terms of certain euclidean three dimensional conformal theories. The first one is the specific holographic relation

$$
m_{\sigma}^{2} L_{d S}^{2}=2 \delta(3-2 \delta)
$$

between the $4 \mathrm{D}$ de Sitter scale $L_{d S}^{2}$ and $4 \mathrm{D}$ scalar field mass $m_{\sigma}$ with the anomalous scaling dimensions $\Delta_{\sigma}=2 \delta$ of the perturbing relevant operators with $2 \delta<3$ in the corresponding $\mathrm{CFT}_{3}$. The second one concerns the value of the central charges

$$
\mathbf{a}_{E}=S_{\Lambda}=4 \pi^{2} \frac{L_{d S}^{2}}{l_{p l}^{2}}=\mathbf{c}_{E}
$$

of these crossovers euclidean $\mathrm{CFT}_{3}$ 's, directly related to the asymptotic limit of the corresponding horizons entropy [48] or its GB improved version [47-49]:

$$
\mathbf{a}_{G B}(j)=S_{0 j}+S_{\Lambda}=\frac{4 \pi^{2} L_{d S}^{2}}{l_{p l}^{2}}\left(1+\frac{2|\lambda| L_{j}^{2}}{L_{d S}^{2}}\right),
$$

but now with $\mathbf{a}_{G B} \neq \mathbf{c}_{G B}$ (see [48,64]). According to our discussion in section 5.3 above, the values of the central charges $\mathbf{a}_{G B}(j)$ of the sequence of $C F T_{3}\left(\mathcal{X}_{j}\right)$ are determined by the specific choice of the GB scales $L_{j}$ at different aeons $\mathcal{A}_{j}$, related to the conditions imposed on the entropy jumps or by requiring its continuity on each crossover (when it is possible). For example, in the case of equal GB scales $L_{j}=L \propto l_{p l}$ all the crossovers are represented by a unique $C F T_{3}$, which in the CCC framework corresponds to the complete loss of the entropy $S_{\Lambda}$ created at the precedent cycle. No primordial crossover needs to be introduced in this case, and indeed the evolution of such SFD symmetric CCC model turns out to be completely cyclic.

\footnotetext{
${ }^{25}$ Which might contain Weyl gravity or/and the conformal coupling of a scalar field to Einstein gravity, with conformal invariant self-interaction.

${ }^{26}$ And their off-critical (perturbed $\left.C F T_{3}\right) /\left(\right.$ asymptotic $\left.d S_{4}\right)$ version [62-64].
} 
We should mention in conclusion that our partially self-dual CCC-like models also provide all the remaining ingredients needed for the complete realization of their holographic description in terms of the "RG flows" in the dual perturbed $C F T_{3}$ [62-64], namely their exact beta functions, the explicit form of the corresponding relevant (and irrelevant) 3-d operators and finally - the non-perturbative form of their correlation functions that can be used for the holographic reconstruction of the density fluctuations spectrum of such CCC-like cosmological models.

\section{Acknowledgments}

ALAL thanks CAPES (Brazil) for financial support. The research of UCdS is partially supported by FAPES(Brazil).

\section{A Cyclic SFD models}

Here we give an explicit example of the construction of a chain of dual aeons described in section 2, representing a cyclic evolution of an universe with deceleration and acceleration periods and with a finite conformal-time lifespan. A specially appropriate case is the Chaplygin gas (3.2), with $\delta=1 / 2$. As stated in section 4, it satisfies the SRM hypothesis. Also, it is partially self-dual, as described in section 3.1. The scale factor evolution may be readily integrated:

$$
a_{1}\left(\eta_{1}\right)=\left(\frac{\rho_{r 1}}{\rho_{\Lambda 1}}\right)^{\frac{1}{4}} \tan \left[\frac{\pi}{2} \eta_{1} / \eta_{f}\right] .
$$

where $\eta_{f}=\frac{3 \pi}{2\left(\rho_{r 1} \rho_{\Lambda 1}\right)^{1 / 4}}$. This aeon, $\mathcal{A}_{1}$, has a big-bang at $\eta=0$ and ends at $\eta=\eta_{f}$, so $\eta_{1} \in\left[0, \eta_{f}\right]$. We may use eq. (2.10) to find an adjacent aeon, either $\mathcal{A}_{0}$ or $\mathcal{A}_{2}$. Let us choose the former, with $a_{0}\left(\eta_{0}\right)=c_{0}^{2} / a_{1}\left(-\eta_{0}\right)$, i.e.

$$
a_{0}\left(\eta_{0}\right)=c_{0}^{2}\left(\frac{\rho_{\Lambda 1}}{\rho_{r 1}}\right)^{\frac{1}{4}} \cot \left[-\frac{\pi}{2} \eta_{0} / \eta_{f}\right]=\left(\frac{\rho_{r 0}}{\rho_{\Lambda 0}}\right)^{\frac{1}{4}} \tan \left[\frac{\pi}{2}\left(\eta_{0}+\eta_{f}\right) / \eta_{f}\right] .
$$

In the last equality, we have used a trigonometric identity, $\cot (-x)=\tan (x+\pi / 2)$, and the relation (3.5) between the parameters. Comparison between this equality and (A.1) illustrates explicitly the partial self-duality of the model: the dual functions $a_{1}\left(\eta_{1}\right)$ and $a_{0}\left(\eta_{1}\right)$ have exactly the same form, the difference being only in the values of the parameters $\left\{\rho_{r 1}, \rho_{\Lambda 1}\right\}$ and $\left\{\rho_{r 0}, \rho_{\Lambda 0}\right\}$.

It is instructive to find now $\mathcal{A}_{-1}$, since according to the $Z_{2}$ property of (2.10) its scale factor must be identical with (A.1), including the same values of the parameters. Indeed, we have

$$
a_{-1}\left(\eta_{-1}\right)=\frac{c_{0}^{2}}{a_{0}\left(-\eta_{-1}-2 \eta_{f}\right)}=\left(\frac{\rho_{r 1}}{\rho_{\Lambda 1}}\right)^{\frac{1}{4}} \tan \left[-\frac{\pi}{2} \frac{\left(\eta_{-1}+2 \eta_{f}\right)}{\eta_{f}}\right]=\left(\frac{\rho_{r 1}}{\rho_{\Lambda 1}}\right)^{\frac{1}{4}} \tan \left[\frac{\pi}{2} \eta_{-1} / \eta_{f}\right]
$$

as expected. In the last equation we have used a property of the periodicity of the $\tan x$ function to demonstrate our point that $a_{-1}\left(\eta_{-1}\right)=a_{1}\left(\eta_{1}\right)$. 


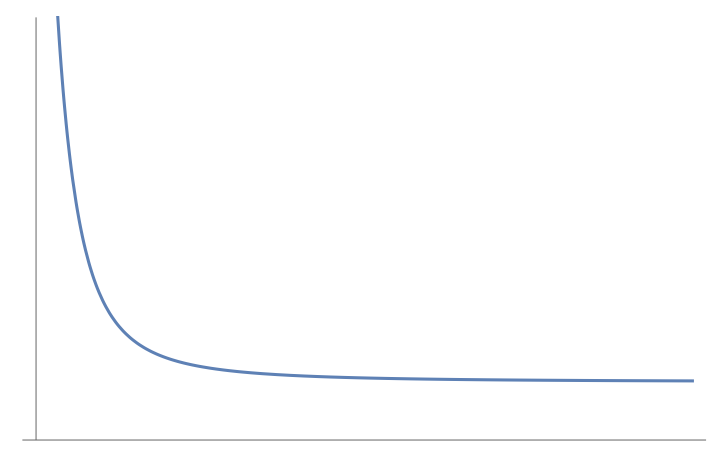

Figure 6. Profile of the function $f(a)$ for $\delta=3 / 4$.

The explicit use of trigonometric identities and the special property of the model being partially self-dual may be misleading, suggesting that our "chain of aeons" could only be constructed for such periodic solutions for the scale factor. This is, of course, not the case. To illustrate that it is not so, consider now a generic scale factor $a_{1}\left(\eta_{1}\right)=g\left(\eta_{1}\right)$, where $g$ is an appropriate function, i.e. $g(0)=0$ e $g\left(\eta_{f}\right)=\infty$ for some $\eta_{f}$. We have

$$
a_{2}\left(\eta_{2}\right)=\frac{c_{0}^{2}}{g\left(-\eta_{2}+2 \eta_{f}\right)}
$$

and

$$
a_{3}\left(\eta_{3}\right)=\frac{c_{0}^{2}}{a_{2}\left(-\eta_{3}+4 \eta_{f}\right)}=g\left(\eta_{3}-2 \eta_{f}\right),
$$

which is the original function with a shifted argument. The important point here is that the domain of $g(x)$ is always the same interval of the real line, namely $x \in\left[0, \eta_{f}\right]$, although the domain of $a_{1}\left(\eta_{1}\right), a_{2}\left(\eta_{2}\right)$, etc. are indeed different from each other. Therefore the "periodicity" of the chain has nothing to do with an eventual periodicity of $g$.

\section{B Proof of the $2^{\text {nd }}$ law for modified Chaplygin gas models}

The $2^{\text {nd }}$ law for the generalized entropy inside the apparent horizon in a universe filled with a modified Chaplygin gas (3.2) is guaranteed within the range of validity of the inequality (5.13) only. Clearly it is equivalent to the positivity of the term in the brackets. Let us denote this term by

$$
f(a)=1+\frac{\sqrt{3} \mathcal{S}}{8 \pi \rho_{r}^{\delta}} \frac{\left(\rho_{r}^{\delta}-\rho_{\Lambda}^{\delta} a^{4 \delta}\right)}{a^{3}\left(\rho_{\Lambda}^{\delta}+\rho_{r}^{\delta} a^{-4 \delta}\right)^{\frac{1}{2 \delta}}} .
$$

We will be concerned only with the physically reasonable range of $\delta$, considered in section 5 , viz. $\frac{3}{4} \leq \delta \leq 1$. We must have $f(a)>0$ for all $a>0$. We first take the limit $a \rightarrow \infty$ and realize that $f>0$ is equivalent to $a^{4 \delta-3}<\frac{8 \pi \rho_{r}^{\delta}}{\sqrt{3} \mathcal{S} \rho_{\Lambda}^{\delta}}$. This inequality is however violated for sufficiently large values of $a$ whenever $\delta>\frac{3}{4}$. Therefore the only possibility that remains is $\delta=\frac{3}{4}$. In this case, it is immediate to verify that $f(a)$ is a monotonic decreasing function, 
cf. figure 6 . The asymptotic inequality

$$
1<\frac{8 \pi \rho_{r}^{3 / 4}}{\sqrt{3} \mathcal{S} \rho_{\Lambda}^{3 / 4}},
$$

(again for $a \rightarrow \infty$ ) becomes identical to the stated one (5.14), once one takes into account the Steffan-Boltzmann law to write $\mathcal{S}=\frac{4}{3}(4 \sigma)^{1 / 4} \rho_{r}^{3 / 4}$ (cf. eq. (5.4)).

Open Access. This article is distributed under the terms of the Creative Commons Attribution License (CC-BY 4.0), which permits any use, distribution and reproduction in any medium, provided the original author(s) and source are credited.

\section{References}

[1] Planck collaboration, P.A.R. Ade et al., Planck 2015 results. XX. Constraints on inflation, Astron. Astrophys. 594 (2016) A20 [arXiv: 1502.02114] [INSPIRE].

[2] G. 't Hooft, Local conformal symmetry: the missing symmetry component for space and time, arXiv:1410.6675 [INSPIRE].

[3] G. 't Hooft, Singularities, horizons, firewalls and local conformal symmetry, arXiv: 1511.04427 [INSPIRE].

[4] K. Hinterbichler and J. Khoury, The pseudo-conformal universe: scale invariance from spontaneous breaking of conformal symmetry, JCAP 04 (2012) 023 [arXiv:1106.1428] [INSPIRE].

[5] C. Csáki, N. Kaloper, J. Serra and J. Terning, Inflation from broken scale invariance, Phys. Rev. Lett. 113 (2014) 161302 [arXiv:1406.5192] [INSPIRE].

[6] I. Bars, P. Steinhardt and N. Turok, Local conformal symmetry in physics and cosmology, Phys. Rev. D 89 (2014) 043515 [arXiv: 1307.1848] [INSPIRE].

[7] R. Kallosh, A. Linde and T. Rube, General inflaton potentials in supergravity, Phys. Rev. D 83 (2011) 043507 [arXiv: 1011.5945] [INSPIRE].

[8] S. Kachru, R. Kallosh, A.D. Linde and S.P. Trivedi, De Sitter vacua in string theory, Phys. Rev. D 68 (2003) 046005 [hep-th/0301240] [INSPIRE].

[9] R. Kallosh and A. Linde, Universality class in conformal inflation, JCAP 07 (2013) 002 [arXiv: 1306.5220] [INSPIRE].

[10] R. Kallosh, A. Linde and D. Roest, Large field inflation and double $\alpha$-attractors, JHEP 08 (2014) 052 [arXiv: 1405.3646] [INSPIRE].

[11] R. Kallosh and A. Linde, Escher in the Sky, Comptes Rendus Physique 16 (2015) 914 [arXiv: 1503.06785] [INSPIRE].

[12] S. Ferrara, R. Kallosh, A. Linde and M. Porrati, Minimal supergravity models of inflation, Phys. Rev. D 88 (2013) 085038 [arXiv: 1307.7696] [INSPIRE].

[13] S. Ferrara, R. Kallosh, A. Linde, A. Marrani and A. Van Proeyen, Superconformal symmetry, NMSSM and inflation, Phys. Rev. D 83 (2011) 025008 [arXiv: 1008.2942] [INSPIRE].

[14] G. Veneziano, Scale factor duality for classical and quantum strings, Phys. Lett. B 265 (1991) 287. 
[15] M. Gasperini and G. Veneziano, The pre-big bang scenario in string cosmology, Phys. Rept. 373 (2003) 1 [hep-th/0207130] [INSPIRE].

[16] J. Khoury, B.A. Ovrut, P.J. Steinhardt and N. Turok, The ekpyrotic universe: colliding branes and the origin of the hot big bang, Phys. Rev. D 64 (2001) 123522 [hep-th/0103239] [INSPIRE].

[17] R. Penrose, Cycles of time: an extraordinary new view of the universe, Random House, U.S.A. (2010).

[18] P.J. Steinhardt and N. Turok, Cosmic evolution in a cyclic universe, Phys. Rev. D 65 (2002) 126003 [hep-th/0111098] [INSPIRE].

[19] A.D. Linde, Inflationary theory versus ekpyrotic/cyclic scenario, talk give at the Conference on the Future of Theoretical Physics and Cosmology in Honor of Steven Hawking's $60^{\text {th }}$ birthday, January 7-10, Cambridge, U.K. (2002), hep-th/0205259 [INSPIRE].

[20] J. Khoury, B.A. Ovrut, N. Seiberg, P.J. Steinhardt and N. Turok, From big crunch to big bang, Phys. Rev. D 65 (2002) 086007 [hep-th/0108187] [INSPIRE].

[21] G.N. Felder, A.V. Frolov, L. Kofman and A.D. Linde, Cosmology with negative potentials, Phys. Rev. D 66 (2002) 023507 [hep-th/0202017] [InSPIRE].

[22] A. Giveon, M. Porrati and E. Rabinovici, Target space duality in string theory, Phys. Rept. 244 (1994) 77 [hep-th/9401139] [INSPIRE].

[23] U. Camara da Silva, A.A. Lima and G.M. Sotkov, Scale factor self-dual cosmological models, JHEP 07 (2015) 017 [arXiv: 1502.06497] [INSPIRE].

[24] M.P. Dabrowski, T. Stachowiak and M. Szydłowski, Phantom cosmologies, Phys. Rev. D 68 (2003) 103519 [hep-th/0307128] [INSPIRE].

[25] L.P. Chimento and R. Lazkoz, On the link between phantom and standard cosmologies, Phys. Rev. Lett. 91 (2003) 211301 [gr-qc/0307111] [INSPIRE].

[26] L.P. Chimento and W. Zimdahl, Duality invariance and cosmological dynamics, Int. J. Mod. Phys. D 17 (2008) 2229 [gr-qc/0609104] [InSPIRE].

[27] M. Gasperini and G. Veneziano, Inflation, deflation and frame independence in string cosmology, Mod. Phys. Lett. A 8 (1993) 3701 [hep-th/9309023] [INSPIRE].

[28] U.d. Camara, C.P. Constantinidis, A.L.A. Lima and G.M. Sotkov, Self-duality from new massive gravity holography, JHEP 05 (2013) 013 [arXiv: 1302.5795] [INSPIRE].

[29] U. Debnath, A. Banerjee and S. Chakraborty, Role of modified Chaplygin gas in accelerated universe, Class. Quant. Grav. 21 (2004) 5609 [gr-qc/0411015] [INSPIRE].

[30] H.B. Benaoum, Accelerated universe from modified Chaplygin gas and tachyonic fluid, hep-th/0205140 [INSPIRE].

[31] J. Lu, D. Geng, L. Xu, Y. Wu and M. Liu, Reduced modified Chaplygin gas cosmology, JHEP 02 (2015) 071 [arXiv: 1312.0779] [inSPIRE].

[32] V.G. Gurzadyan and R. Penrose, On CCC-predicted concentric low-variance circles in the CMB sky, Eur. Phys. J. Plus 128 (2013) 22 [arXiv:1302.5162] [INSPIRE].

[33] E. Newman, A fundamental solution to the CCC equations, Gen. Rel. Grav. 46 (2014) 1717 [arXiv: 1309.7271] [INSPIRE].

[34] P. Tod, The equations of CCC, arXiv:1309.7248 [INSPIRE]. 
[35] R. Kallosh, Planck 2013 and superconformal symmetry, arXiv:1402.0527 [INSPIRE].

[36] K.A. Meissner and P. Nurowski, Conformal transformations and the beginning of the Universe, arXiv: 1506.03280 [INSPIRE].

[37] M. Gasperini and G. Veneziano, Pre-big bang in string cosmology, Astropart. Phys. 1 (1993) 317 [hep-th/9211021] [INSPIRE].

[38] G.W. Gibbons and S.W. Hawking, Cosmological event horizons, thermodynamics, and particle creation, Phys. Rev. D 15 (1977) 2738.

[39] R.M. Wald, Black hole entropy is the Noether charge, Phys. Rev. D 48 (1993) R3427 [gr-qc/9307038] [INSPIRE].

[40] R. Bousso and N. Engelhardt, Generalized second law for cosmology, Phys. Rev. D 93 (2016) 024025 [arXiv: 1510.02099] [INSPIRE].

[41] R. Bousso and N. Engelhardt, New area law in general relativity, Phys. Rev. Lett. 115 (2015) 081301 [arXiv: 1504.07627] [INSPIRE].

[42] S. Weinberg, Gravitation and cosmology: principles and applications of the general theory of relativity, volume 1, Wiley, New York U.S.A. (1972).

[43] T. Jacobson, Thermodynamics of space-time: the Einstein equation of state, Phys. Rev. Lett. 75 (1995) 1260 [gr-qc/9504004] [INSPIRE].

[44] G.M. Sotkov, A.L.A. Lima and U. Camara da Silva, On the thermodynamics of scale factor dual Universes, arXiv:1609.00393 [INSPIRE].

[45] R.-G. Cai and S.P. Kim, First law of thermodynamics and Friedmann equations of Friedmann-Robertson-Walker universe, JHEP 02 (2005) 050 [hep-th/0501055] [INSPIRE].

[46] M. Akbar and R.-G. Cai, Thermodynamic behavior of Friedmann equations at apparent horizon of FRW universe, Phys. Rev. D 75 (2007) 084003 [hep-th/0609128] [INSPIRE].

[47] R.-G. Cai and Y.S. Myung, Holography and entropy bounds in Gauss-Bonnet gravity, Phys. Lett. B 559 (2003) 60 [hep-th/0210300] [INSPIRE].

[48] A. Sinha, On higher derivative gravity, c-theorems and cosmology, Class. Quant. Grav. 28 (2011) 085002 [arXiv: 1008.4315] [INSPIRE].

[49] U. Camara dS, A.A. Lima and G.M. Sotkov, Energy density bounds in cubic quasi-topological cosmology, arXiv:1304.4185 [INSPIRE].

[50] R. Brandenberger and P. Peter, Bouncing cosmologies: progress and problems, arXiv: 1603.05834 [INSPIRE].

[51] R. Kallosh, J. U. Kang, A. Linde and V. Mukhanov, The new ekpyrotic ghost, JCAP 04 (2008) 018 [arXiv:0712.2040].

[52] S.D. Hsu, A. Jenkins and M.B. Wise, Gradient instability for $w<-1$, Phys. Lett. B 597 (2004) 270 [INSPIRE].

[53] V.A. Belinskii, I.M. Khalatnikov and E.M. Lifshitz, Oscillatory approach to a singular point in the relativistic cosmology, Adv. Phys. 19 (1970) 525.

[54] T. Damour, M. Henneaux and H. Nicolai, Cosmological billiards, Class. Quant. Grav. 20 (2003) R145 [hep-th/0212256] [INSPIRE].

[55] J.-L. Lehners, Ekpyrotic and cyclic cosmology, Phys. Rept. 465 (2008) 223 [arXiv:0806.1245] [INSPIRE]. 
[56] E. I. Buchbinder, J. Khoury and B.A. Ovrut, New ekpyrotic cosmology, Phys. Rev. D 76 (2007) 123503 [hep-th/0702154] [INSPIRE].

[57] S.M. Carroll, M. Hoffman and M. Trodden, Can the dark energy equation-of-state parameter $w$ be less than -1?, Phys. Rev. D 68 (2003) 023509 [astro-ph/0301273] [InSPIRE].

[58] R. Brustein, M. Gasperini and G. Veneziano, Duality in cosmological perturbation theory, Phys. Lett. B 431 (1998) 277 [hep-th/9803018] [INSPIRE].

[59] I. Antoniadis, P.O. Mazur and E. Mottola, Conformal invariance and cosmic background radiation, Phys. Rev. Lett. 79 (1997) 14 [astro-ph/9611208] [INSPIRE].

[60] I. Antoniadis, P.O. Mazur and E. Mottola, Conformal Invariance, Dark Energy and CMB Non-Gaussianity, JCAP 09 (2012) 024 [arXiv:1103.4164] [INSPIRE].

[61] M. Libanov, V. Rubakov and G. Rubtsov, Towards conformal cosmology, JETP Lett. 102 (2015) 561 [arXiv: 1508.07728] [inSPIRE].

[62] F. Larsen, J.P. van der Schaar and R.G. Leigh, De Sitter holography and the cosmic microwave background, JHEP 04 (2002) 047 [hep-th/0202127] [INSPIRE].

[63] J.P. van der Schaar, Inflationary perturbations from deformed CFT, JHEP 01 (2004) 070 [hep-th/0307271] [INSPIRE].

[64] P. McFadden and K. Skenderis, The holographic universe, J. Phys. Conf. Ser. 222 (2010) 012007 [arXiv: 1001.2007] [INSPIRE].

[65] A. Strominger, The dS/CFT correspondence, JHEP 10 (2001) 034 [hep-th/0106113] [INSPIRE].

[66] A. Strominger, Inflation and the dS/CFT correspondence, JHEP 11 (2001) 049 [hep-th/0110087] [INSPIRE]. 